\title{
A production-based model for a fractured well in unconventional reservoirs
}

\author{
Shaibu Mohammed ${ }^{1}$, Prosper Anumah ${ }^{1}$, Justice Sarkodie-kyeremeh ${ }^{1}$, and Emmanuel \\ Acheaw 1
}

${ }^{1}$ Department of Energy and Petroleum Engineering, University of Energy and Natural Resources, Sunyani, Ghana.

\begin{abstract}
Due to the depletion of conventional reservoirs and the high demand of energy, unconventional reservoirs will be relied on to supply the world's energy for the foreseeable future. Unfortunately, modelling and analysis of these reservoirs have been very challenging because of their complex storage and flow mechanisms. Although analytical, semi-analytical and numerical models have been proposed, these models rely on simplifying assumptions and require several input parameters.

In this paper, a production-based model is proposed to analyze and predict a fractured-well performance in unconventional reservoirs. The model assumes a power law with a stretched exponential cut-off. While the power-law term governs the transient-state period, the stretched exponential term, which is a superposition of exponential decays, governs the boundary-dominated flow period. As a result, the model is capable of matching both the transient state and boundarydominated flow portions of the data.

The model has been validated with a numerical data and applied to several field data; in addition, the model has been used to estimate P10, P50 and P90 values, as well as to develop P10, P50 and P90 type curves for the Barnett shale. These type curves will be useful for production forecasting of new wells in the field or analogue fields. Results of the model have been compared with existing models. The findings show that the proposed model yields relatively good reserve estimates, and predicts the future production performance of unconventional reservoirs not only during the transient-state period, but also the boundary-dominated flow period. The proposed model may contribute to the ongoing efforts to improve the analysis and forecasting of fracturedwell performance in unconventional reservoirs.
\end{abstract}

Keywords: Unconventional reservoirs; Fractured-well performance; Production-based model; Reserve estimate; P90, P50 and P10 type curves; Barnett shale.

\subsection{Introduction}

Although renewable energy has gained impetus, fossil fuels will largely be used as energy for the foreseeable future. Fossil fuels from unconventional reservoirs (such shales, tight sandstones and coalbed methane) have heavily been explored and exploited for decades. This is mainly attributed to the advent of horizontal well drilling and hydraulic fracturing technology. Other reasons are the depletion of conventional reservoirs and the high energy demand.

As opposed to conventional reservoirs, unconventional reservoirs have complex storage mechanisms due to their different sites for the free and adsorbed hydrocarbons, and complex flow mechanisms due to their varying pore sizes and tortuosity (Javadpour 2007). Furthermore, due to the extremely low permeability of these reservoirs, Knudsen diffusion, instead of Darcy flow, 
prevails in the nanopores; also, the stress sensitivity of these reservoirs precludes the use of intrinsic permeability. Natural and induced fractures networks add to the complexity of these reservoirs too. In addition, depending on the hydraulic fracture spacing and the extent of the enhanced permeability of the stimulated reservoir volume, unconventional reservoirs may experience a fracture interference in the life time of a well, causing a no-flow boundary between adjacent hydraulic fractures (Joshi and Lee 2013). As a result, even though transient-flow regime prevails for long periods in low-permeability reservoirs (Wattenbarger et al. 1998), boundarydominated flow regime can be observed prior to abandonment (Male 2019). Due to these complexities, modelling and analysis of unconventional reservoirs have been very challenging.

Ozkan et al. (2009) and Brown et al. (2009) presented a trilinear flow model to analyze a multifractured horizontal well in unconventional reservoirs. The model accounts not only for fluid flow in the stimulated reservoir volume, but also considers fluid flow from the unstimulated reservoir volume. Other models consider homogeneous formation (Wattenbarger et al. 1998; Wattenbarger 2007; Patzek et al. 2013; Mohammed and Enty 2013; Damoah et al. 2015), dual-porosity formation (Bump and McKee 1988, Passey et al. 2010; El-Banbi 1998; Bello and Wattenbarger 2008; Bello and Wattenbarger 2010; Bello 2009; Al-Ahmadi et al. 2010), triple-porosity formation (AlAhmadi 2011), and simultaneous fluid flow in the matrix (Ezulike and Dehghanpour 2013; Ezulike and Dehghanpour 2015). Numerical models (Moridis et al. 2010; Mirzaei and Cipolla 2012; Yu and Yao 2013; Xue et al. 2018) have also been applied to analyze and forecast these reservoirs. Although these models (analytical, semi-analytical and numerical models) allow the impacts of formation and fractures properties on reservoir performance to be ascertained, several input parameters are involved, some of which are not readily available. Furthermore, analytical and semi-analytical models rely on somewhat simplifying assumptions, which may not be realistic. Consequently, some researchers (Raghavan 2011; Raghavan and Chen 2013a and 2013b; Ozcan et al. 2014; Zou et al. 2016) have argued that anomalous or fractional diffusion, instead of normal diffusion, may be well suited for unconventional reservoirs.

Production-based models have also been presented to analyze unconventional reservoir performance (Ilk et al. 2008; Valko 2009; Valko and Lee 2010; Clark et al. 2011; Duong 2011; Wang et al. 2017; Miao et al 2018; Miao et al 2020). Arps (1945) developed an empirical model that has widely been used to analyze and forecast well performance. For unconventional reservoirs, however, the transient-state period may persist for several years (Wattenbarger et al. 1998; Duong 2011), and the Arps's model may be unsuitable. As a result, values of the hyperbolic decline exponent greater than unity have been used to analyze unconventional reservoir performance; unfortunately, this approach yields unrealistic estimates during the boundary-dominated flow period (Lee and Sidle 2010; Duong 2011). Ilk et al. (2008) presented a power-law exponential (PLE) model. The PLE model is capable of matching both the transient-state and boundarydominated flow periods; however, the PLE model requires that the data is transformed via a differentiation algorithm, which may amplify the noise inherent in production data. Clark et al. (2011) proposed a logistic growth model (LGM) to analyze and forecast unconventional reservoir performance. The LGM is based on the premise that, due to resource constraint, growth is finite. Duong (2011) proposed an empirical model for fracture-dominated unconventional reservoirs. His model is suitable for transient linear and bilinear flow, but has proven to be unsatisfactory during 
the boundary-dominated flow period (Kanfar and Wattenbarger 2012); furthermore, during the early-time period, the Duong's model exhibits a "false" incline phase.

In this paper, a production-based model is proposed for the analysis and forecasting of a fractured-well performance in unconventional reservoirs. The model has been developed based on a modified version of the Duong's model. The objectives are to alleviate the "artificial" incline phase exhibited by the Duong's model, and impose a constraint during the boundary-dominated flow period.

The main body of the paper is organized as follows: First, the inherent drawbacks of Duong's model are presented. Second, a production-based model, which is a modified version of the Duong's model, is proposed as an attempt to address these drawbacks. Finally, the model is validated with a simulated data, and applied to several field data.

\subsection{Duong's model}

In theory, the response of a fractured well in unconventional reservoir obeys a power-law model during the transient-state period (Wattenbarger et al. 1998; Al Ahmadi et al. 2010):

$$
q=q_{1}\left(\frac{t}{\tau}\right)^{-n}
$$

where $q$ is the flow rate (Mscf/D or STB/D); $t$ and $\tau$ are the time and characteristic time, respectively, in days or months or years; $q_{1}$ is the flow rate at $t / \tau=1$; and $n$ is the decline exponent (dimensionless), which governs a given flow regime. Integrating Equation 1 with respect to time yields:

$$
Q=\frac{q_{1} \tau^{n}}{1-n} t^{1-n}
$$

Combining Equations 1 and 2 gives:

$$
\frac{q}{Q}=\frac{1-n}{t}
$$

Equation 3 suggests that a $\log -\log$ plot of $q / Q$ against $t$ will yield a straight-line of slope, -1 , and an intercept, $(1-n)$. Duong (2011) observed that field data do not lend themselves to Equation 3; thus, Equation 3 is not well suited for a fractured well in unconventional reservoirs. As a consequence, and based on field data, Duong (2011) adjusted Equation 3 to give:

$$
\frac{q}{Q}=a t^{-m}
$$

Although Equation 4 approximates the performance of a fractured well in unconventional reservoirs quite well, it has at least two drawbacks. These drawbacks are apparent if one rearranges Equation 4 to yield:

$$
q=a t^{-m} Q
$$

which is, fundamentally, the production-based model proposed by Duong (2011). Cumulative production, $Q$, increases with time, while the power-law term, $t^{-m}$, decreases with time. Thus, the first drawback emanates from the cumulative production term in Equation 5. This is because as 
cumulative production increases with time, it forces the Duong's model to exhibit an incline phase at early times; unfortunately, this incline phase is false as it does not model the actual incline phase observed in field data. The second drawback is due to the power-law term. This is because while the late-time data obeys an exponential decline (Ilk et al. 2008; Kanfar and Wattenbarger 2012), the power-law term forces the Duong's model to assume a power law, instead of an exponential decay, during the late-time period. These drawbacks can further be deduced from the cumulative and rate models given in Equations 6 and 7, respectively (Duong 2011):

$$
\begin{aligned}
& Q=\frac{q_{1}}{a} e^{\left[\frac{a}{1-m}\left(t^{1-m}-1\right)\right]} \\
& q=q_{1} t^{-m} e^{\left[\frac{a}{1-m}\left(t^{1-m_{-1}}\right)\right]}
\end{aligned}
$$

It can be seen that Equation 6 has been substituted into Equation 5 to yield Equation 7. Thus, the exponential term in Equation 7 is attributed to the cumulative production, which increases with time; and, as a result, causes the Duong's model to exhibit a false incline phase at early time. Figure 1 shows rate-decline type curves based on the Duong's model (Equation 7). The curves rise and reach a maximum, and then decline. The incline phase is due to the cumulative production term, and hence an artefact introduced by the Duong's Model. Also, because the decline phase is governed by the power-law portion, $t^{-m}$, the Duong's model fails to match the boundarydominated flow data, which is governed by an exponential decay (Ilk et al. 2008; Kanfar and Wattenbarger 2012; Gupta et al. 2019). Thus, the Duong's model is strictly applicable to the transient-state period.

In the next section, we modify Equation 4, and present a new production-based model that overcomes these drawbacks; and, more importantly, impose a bound during the boundarydominated flow period.

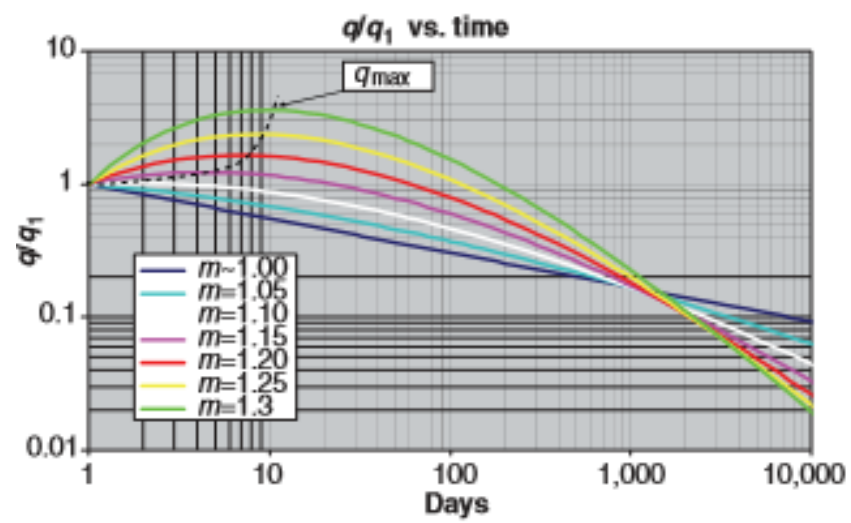

Figure 1: A log-log plot of dimensionless rate against time using the Duong's model (Equation 7). (The Figure has been taken from Duong (2011)). For all values of $m$, except $m=1$ which is uncharacteristic of an unconventional reservoir, the curves incline to a maximum, and then decline. The incline phase is an artefact introduced by the Duong's model. Also, note that even at $\mathrm{t}=10,000$ days, the curves are still linear, which is governed by the power-law term. 


\subsection{Method}

\subsection{Model formulation}

In this section, a production-based model, which is essentially a truncated power-law model, is proposed. The model seeks to overcome the limitations of the Duong's model.

We start with a modified version of the Duong's model (see Equation 4) as:

$$
\frac{q}{K-Q}=a t^{-m}
$$

where $K$ is the technically recoverable resource, or the carrying capacity (Hubbert 1965; Clark et al. 2011), or, as used in this study, the initial fluid-in-place (Male 2019). Thus, essentially, $Q$ has been substituted by $(K-Q)$ in Eq. 4 . This adjustment, as will be shown later, eliminates any "artificial" incline phase, and imposes a bound at late times, thereby avoiding unrealistic predictions at late times. Equation 8 is a kind of a logistic growth model, which is known to impose a bound at late times (Hubbert 1965; Clark et al. 2011). If one re-arranges Equation 8, she/he arrives at:

$$
q=a t^{-m}(K-Q)
$$

As opposed to $Q,(K-Q)$ declines with time; thus, Equation 9 will be more conservative than Equation 5. Now, considering dimensionality, Equation 9 is re-written as:

$$
q=\frac{n}{t}\left(\frac{t}{\tau}\right)^{n}(K-Q)
$$

Primarily, Equation 10 constitutes the proposed production-based model to analyze and predict a fractured-well performance in unconventional reservoirs. Next, we express Equation 10 explicitly as a function of time to allow for analysis and future performance prediction.

Flow rate is related to the derivative of cumulative production as:

$$
q=\frac{d Q}{d t}
$$

Substituting Equation 11 into Equation 10 yields

$$
\frac{d Q}{d t}=\frac{n}{t}\left(\frac{t}{\tau}\right)^{n}(K-Q)
$$

Applying the method of separation of variables to Equation 12, and integrating the result, we have (noting that the initial condition is $Q(t=0)=0$ ):

$$
\int_{0}^{Q} \frac{1}{K-Q} d Q^{\prime}=\frac{n}{\tau^{n}} \int_{0}^{t} t^{-(1-n)} d t^{\prime}
$$

Solving for $Q$, which is the cumulative production, we have:

$$
Q=K\left\{1-\exp \left[-\left(\frac{t}{\tau}\right)^{n}\right]\right\}
$$

The rate model is obtained by differentiating Equation 14 with respect to time (or substituting Equation 14 into Equation 10) to give: 


$$
q=\frac{K}{\tau} n\left(\frac{t}{\tau}\right)^{-(1-n)} \exp \left[-\left(\frac{t}{\tau}\right)^{n}\right]
$$

Equations 15 and 14 complete the formulations of the proposed rate and cumulative productionbased models, respectively. These models are power-law stretched exponential (PLSE) because they depict a power law with a stretched exponential cut-off.

PLSE models have been used for several applications: Barraud et al. (2002) fitted spectra of 24 gamma ray bursts with a PLSE model, and found that the PLSE model uses relatively few input parameters and provides a good fit to the data. Fenner et al. (2003) showed that the behaviour of Mathematical Research Network is consistent with a power law exponential cut-off. Gonzalez et al. (2008) have used PLSE to model human population's mobility patterns. Clauset et al. (2009) indicated that the PLSE model is useful for physical phenomena. PLSE model has also been used to fit data samples of Authors' productivity in Chemistry (Smolinsky 2017).

For the specific case where $n=1$, the PLSE model (Equation 15) reduces to the conventional Arps' exponential model. At early time, when a small amount of fluid has been produced relative to the amount initially in-place such that $Q \ll K$, then, $K-Q \approx K$; as a result, at early time, Equation 10 gives:

$$
q=\frac{K}{\tau} n\left(\frac{t}{\tau}\right)^{-(1-n)}
$$

which is the power-law term in Equation 15. Thus, Equation 16 is applicable at early time; and, during this time, Equations 15 and 16 are equivalent. Figure 2 compares the PLSE model (Equation 15) and the power-law model (Equation 16). During the transient-state period up to about 30 months, both models depict the same path; however, at late times, the curves peel off, and the power-law model gives a higher prediction. Thus, the PLSE model is more conservative. Also, contrary to the Duong's model (see Figure 1), Figure 2 does not display any incline phase; this is attributed to the carrying capacity introduced in Equation 8. Figure 3 is the cumulative production plot. The PLSE cumulative plot has been developed from Equation 14 and the power-law cumulative plot has been constructed from the integral of Equation 16. Figure 3 shows that while the PLSE cumulative curve approaches the carrying capacity at late times, the power-law cumulative line exceeds the carrying capacity. Thus, the carrying capacity imposes a bound on the production data at late times. Giving that the carrying capacity, $\mathrm{K}$, is the initial fluid-in-place, the PLSE is conservative. Perhaps, the major finding in Figures 2 and 3 is that the PLSE model exhibits a power law at early times, and tends to exponential decline at late times. This behaviour is a characteristic of a fractured well completed in low- and ultra-low permeability reservoirs (Wattenbarger et al. 1998; Ilk et al. 2008; Kanfar and Wattenbarger 2011). 


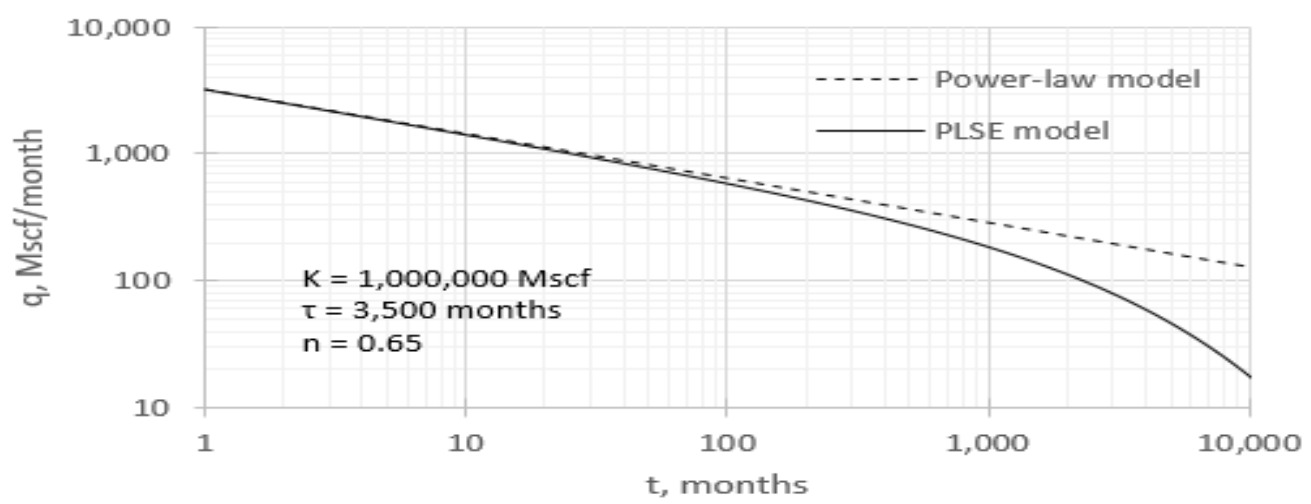

Figure 2: A log-log plot of rate against time for the power-law model and power-law stretched exponential model (PLSE). The models are indistinguishable during the transient-state period, but separate during the boundary-dominated flow period. Clearly, the PLSE model is more conservative.

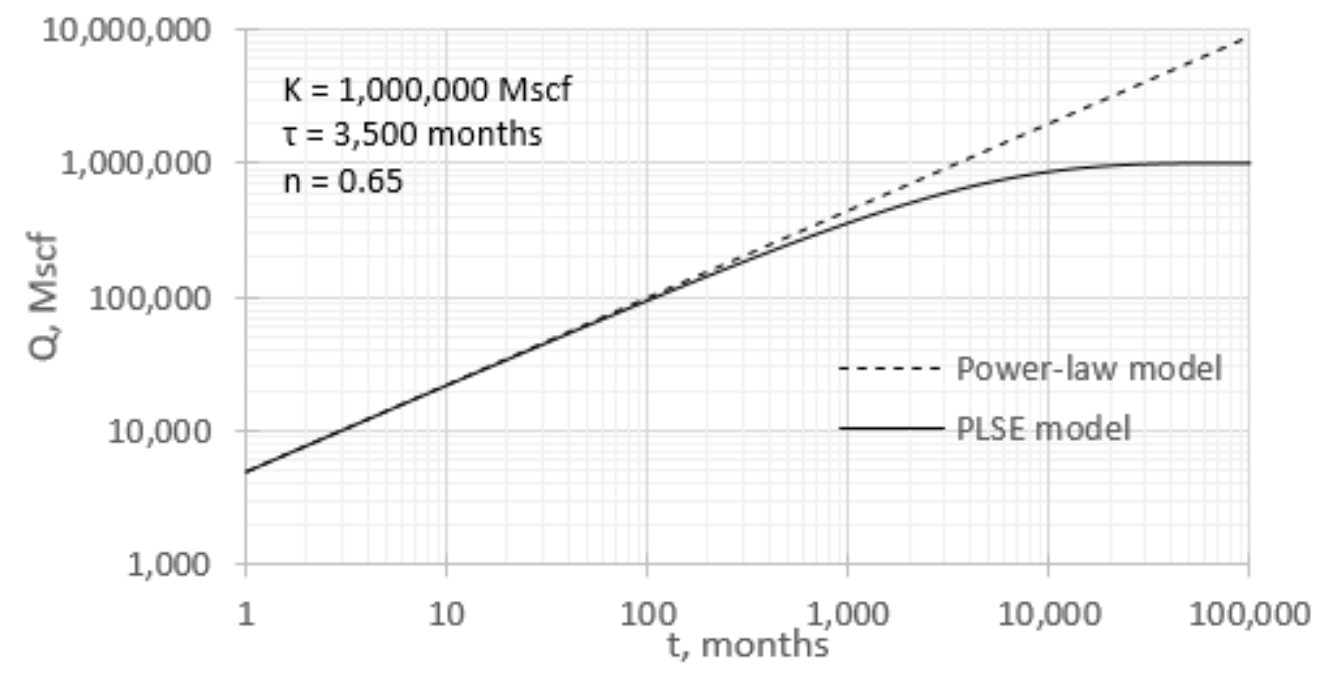

Figure 3: A log-log plot of cumulative production against time for the power-law model and power-law stretched exponential (PLSE) model. The curves are indistinguishable at early times, but separate at late times, with the PLSE curve approaching the value of the carrying capacity.

\subsection{Reserve Estimate}

The estimated ultimate recovery (EUR) can be derived from Equation 14 as:

$$
E U R=K\left\{1-\exp \left[-\left(\frac{t_{\text {eco }}}{\tau}\right)^{n}\right]\right\}
$$

where $t_{e c o}$ is the time at the economic limit (i.e., $t$ at $q=q_{e c o}$, where $q_{e c o}$ is the economic rate).

\subsection{Model parameters estimation}

The PLSE is a three-parameter model. These parameters include the decline exponent, $n$, the characteristic time, $\tau$, and the carrying capacity, $K$. To analyze and predict a fractured-well performance, these parameters must be estimated. 
The cumulative production model (i. e., Equation 14) can be re-written as:

$$
z(K, Q)=\frac{1}{\tau^{n}} t^{n}
$$

where:

$$
z(K, Q)=\ln \left(\frac{K}{K-Q}\right)
$$

Equation 18 suggests that a $\log -\log$ plot of $z(K, Q)$ against $t$ will yield a straight-line of positive slope, $n$, and an intercept, $\left(1 / \tau^{n}\right) ; n$ and $\tau$ are estimated from the slope and intercept, respectively. The function, $z(K, Q)$, requires a prior knowledge of the carrying capacity, $K . K$ can be estimated from volumetric method. However, if $\mathrm{K}$ is not known a priori from volumetric method, nonlinear regression analysis can be used to estimate the model parameters; in this case, initial estimates of the model parameters are required. The initial estimate of $\mathrm{K}$ is obtained by substituting different values of $\mathrm{K}$ to compute $z(K, Q)$ until the data is linearized using Equation 18 . Then, the initial estimates of $n$ and $\tau$ are estimated from the slope and intercept of Equation 18. Finally, nonlinear regression is used to minimize the square of the sum of the residuals. In this study, Microsoft Excel nonlinear Solver has been used for the nonlinear regression analysis.

\subsection{Results and discussion}

\subsection{Model validation}

\subsubsection{Case 1: Simulated data from Carter (1985)}

In this section, the proposed PLSE model is validated. The simulated data has been taken from Carter (1985). We chose this simulated data to validate the proposed model because not only is the raw production data published in a peer-reviewed journal (i.e., the data can readily be assessed and trusted), a fractured well in a low-permeability reservoir was considered. The initial gas-inplace from the simulator was reported as 3,087,000 Mscf.

Figure 4 shows the production history of the simulated data. Clearly, the data encompasses both the transient-state and boundary-dominated flow periods. Figure 5 (green data points) is constructed based on Equation 18. The carrying capacity is 3,087,000 Mscf. The Duong's plot (reddish-brown data points) has been included in Figure 5 for the sake of comparison. The PLSE model performs better than the Duong's model as it linearizes both the transient and boundarydominated flow data points. The input parameters are estimated, and presented in Table 1. The input parameters of the Logistic growth model (LGM) and the conventional Arps' hyperbolic model have been included in Table 1. In Figure 6, a comparison of the performances of the PLSE model, the Duong's model, the LGM and the Arps' hyperbolic model is made. All the models perform well during the transient-state period; however, during the boundary-dominated flow period, the Duong's model and the Arps' hyperbolic model give higher predictions; the LGM performs quite well but yields lower predictions. The PLSE model seems to trace both the transient-state period and boundary-dominated flow period. As a consequence, the PLSE model appears to be well suited for a fractured-well performance in unconventional reservoirs. 


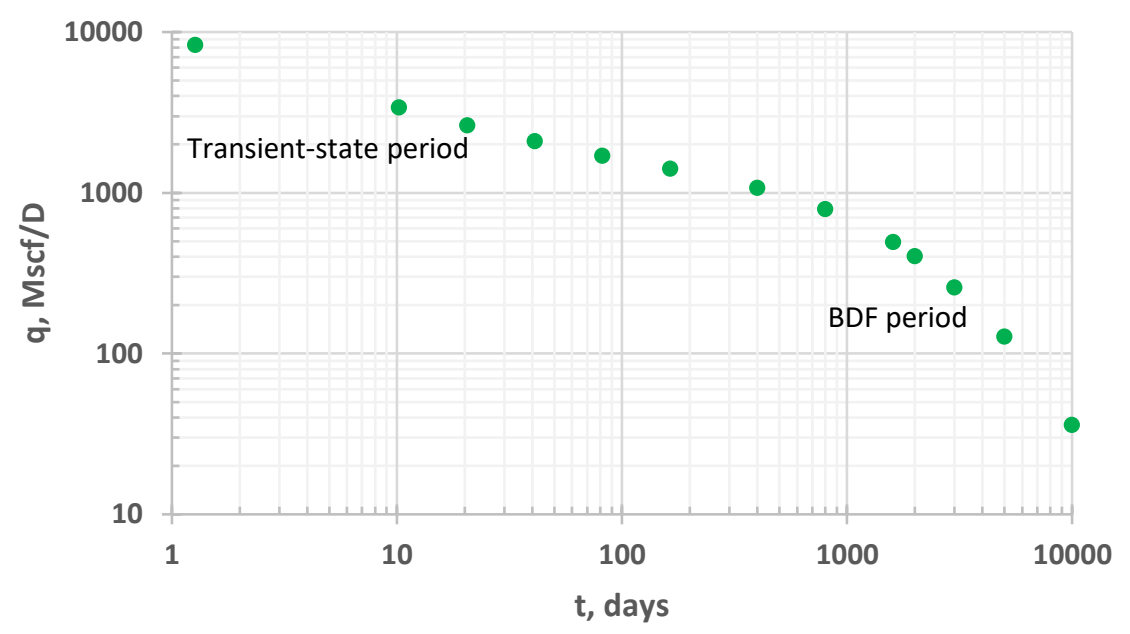

Figure 4: Rate history of the simulated data. The production has been taken from Carter (1985).

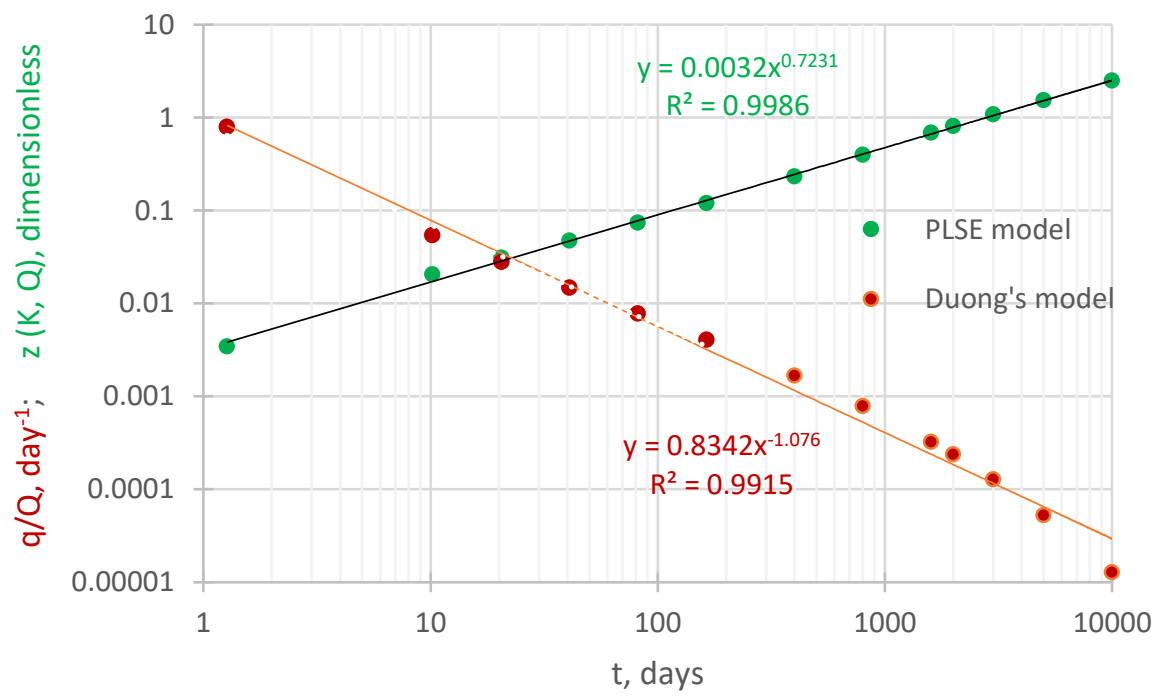

Figure 5: A composite plot of the PLSE model and the Duong's model. It appears that the PLSE model performs better than the Duong's as it linearizes both the transient and boundary-dominated flow data points.

Table 1: Estimated model parameters for case 1

\begin{tabular}{|c|c|c|c|c|c|c|c|c|c|c|c|}
\hline \multicolumn{3}{|c|}{ Duong's model } & \multicolumn{3}{|c|}{ Arps' model } & \multicolumn{3}{|c|}{ PLSE } & \multicolumn{3}{|c|}{ LGM } \\
\hline $\mathrm{q}_{1}$ & $\mathrm{~A}$ & $\mathrm{~m}$ & qi & Di & $\mathrm{b}$ & K & $\tau$ & $\mathrm{n}$ & K & $\mathrm{a}$ & $\mathrm{n}$ \\
\hline 9236 & 1.01 & 1.076 & 550 & 0.000426 & 2 & 3087000 & 2820 & 0.7231 & 3087000 & 500 & 0.8 \\
\hline
\end{tabular}




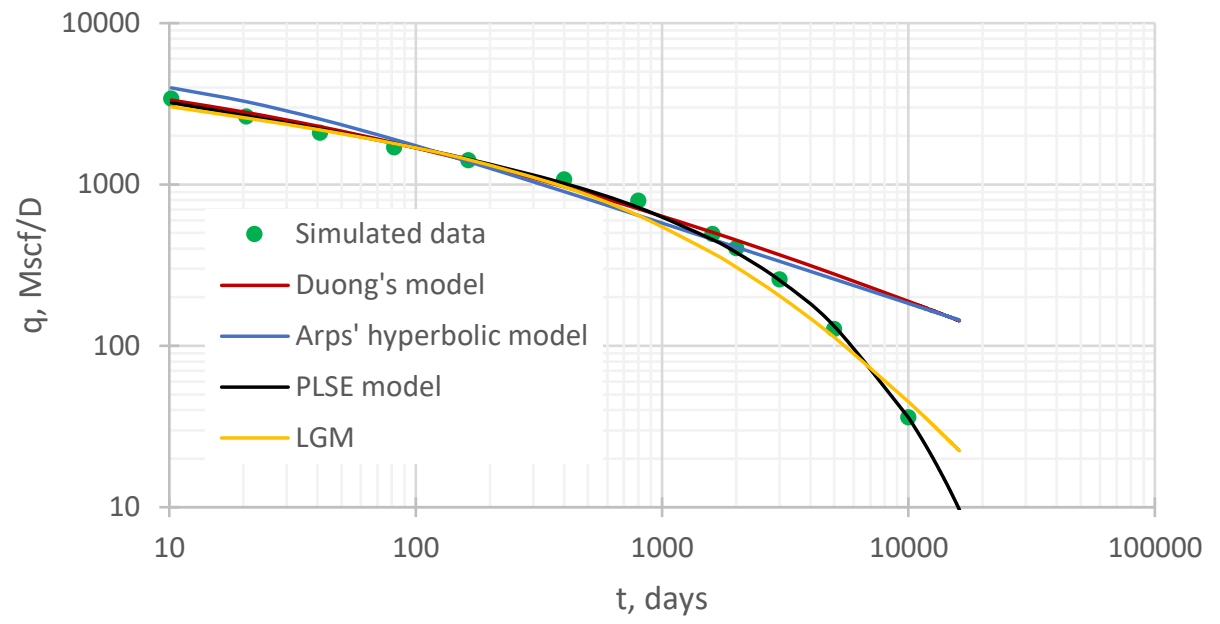

Figure 6: Performance comparison of the PLSE model and other existing models. It appears that the PLSE model performs better than the existing models, especially during the boundarydominated flow period.

\subsection{Sensitivity Analysis}

The model parameters $(n, \tau, K)$ influence the behaviour of the production performance of a fractured well. In this section, the impacts of these parameters on production performance are explored.

\subsubsection{Effect of decline exponent}

The decline exponent characterizes the curvature of the rate-decline curves. Figure 7 shows the effect of the decline exponent on production rate. The decline curves have been developed with Equation 15. Small values of the decline exponent yield higher rates but decline sharply, and the transient state prevails for a long period of time. In contrast, large values of the decline exponent start at low flow rates, and decline steadily; and assume an exponential decline at relatively early times. Figure 8 shows the effect of the decline exponent on the cumulative production. The curves have been developed with Equation 14. At early times, small values of the decline exponent give comparatively high cumulative production; however, at late times, large values of the decline exponent seem to pay off. Note in Figure 8 that regardless of the value of the decline exponent, the curves are bounded by the carrying capacity ( $\mathrm{K}=1,000,000 \mathrm{Mscf})$. These observations may be explained as follows: for small values of the decline exponent, a substantial amount of fluid is withdrawn at early times causing a high reservoir pressure drop. In contrast, for large values of the decline exponent, a small amount of fluid is withdrawn at early times, and so this does not cause a significant reservoir pressure drop. Consequently, for large values of the decline exponent, the reservoir has high pressure to drive the fluid towards the wellbore even at late times; in the case of small values of the decline exponent, the reservoir pressure becomes relatively low, and so it is unable to sustain production at late times. Thus, having a relatively small value of the decline exponent seems to be beneficial because it implies that a substantial amount of fluid can be produced in a relatively short period, and revenue can be generated as early as possible. In contrast, a longer period is required to produce an equivalent amount of fluid if the decline exponent is 
relatively large. Also, although at very large times, the larger decline exponent appears to have a higher cumulative production, the difference is not significant.

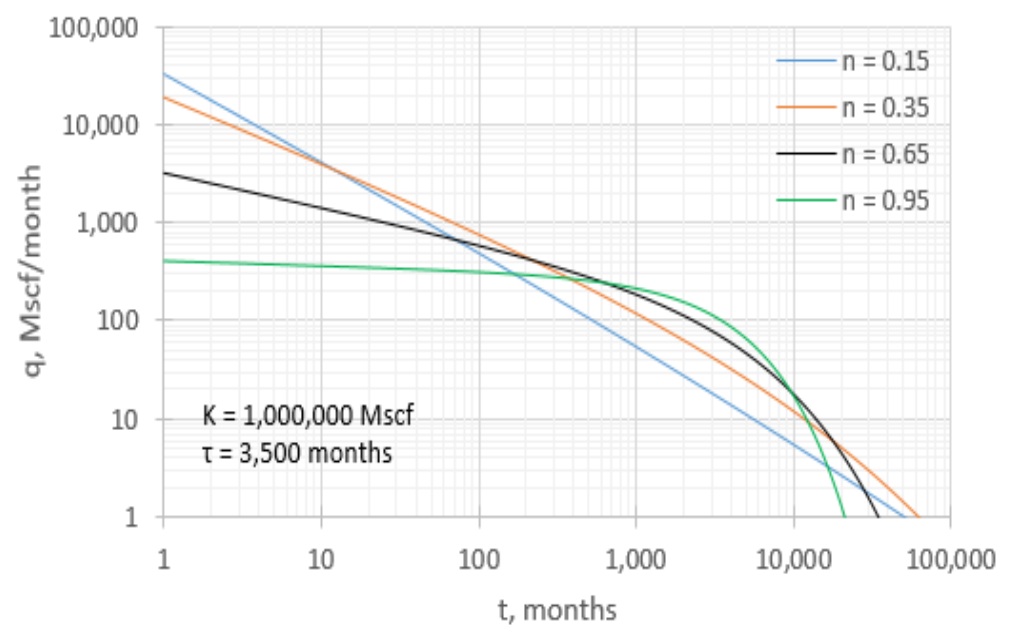

Figure 7: A logarithmic plot of rates against time. The plot is based on Equation 15. Small values of $\mathrm{n}$ yield relatively high rates at early times, but decline sharply, with prolonged straight-lines. Large values of $\mathrm{n}$ yield lower rates with steady decline.

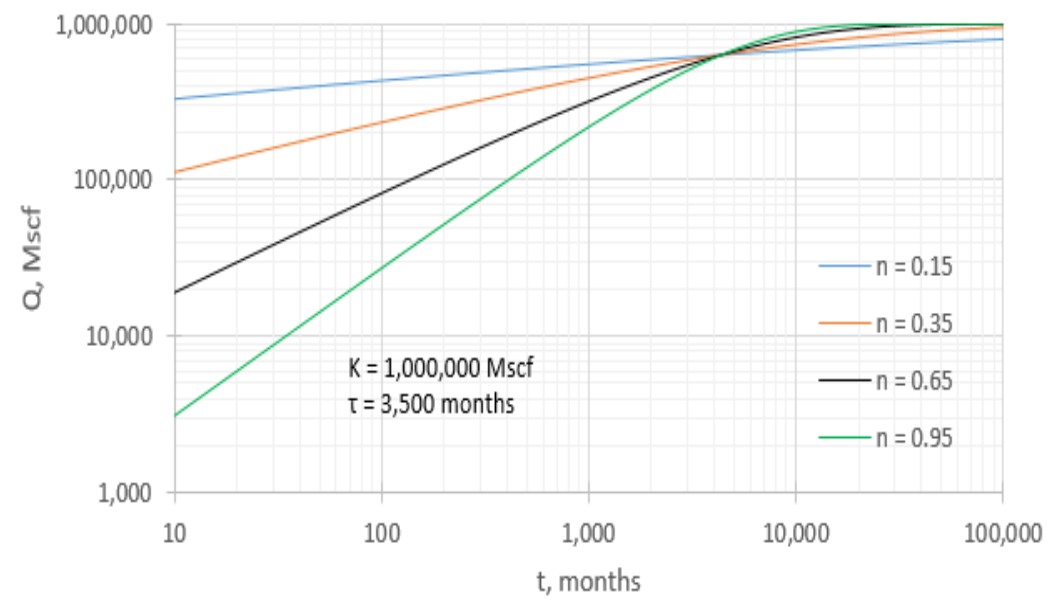

Figure 8: A logarithmic plot of cumulative production against time. The plot is based on Equation 14. Small values of $n$ yield relatively high values at early times, but incline steadily. Large values of $n$ yield lower values at early times, but increases sharply until it approaches the carrying capacity.

\subsubsection{Effect of characteristic time}

Figures 9 and 10 show the effect of characteristic time (tau symbol) on the production performance of a fractured-well performance in unconventional reservoir. In Figure 9, at early times, relatively small values of the characteristic time give higher flow rate. At late time, however, there is a switch, with the relatively large characteristic time registering a higher flow rate. In Figure 10, it is observed that the smaller the characteristic time, the higher the cumulative production; this 
occurs until late times when all the curves converge and approach the carrying capacity. As a consequence, small values of characteristic time is beneficial.

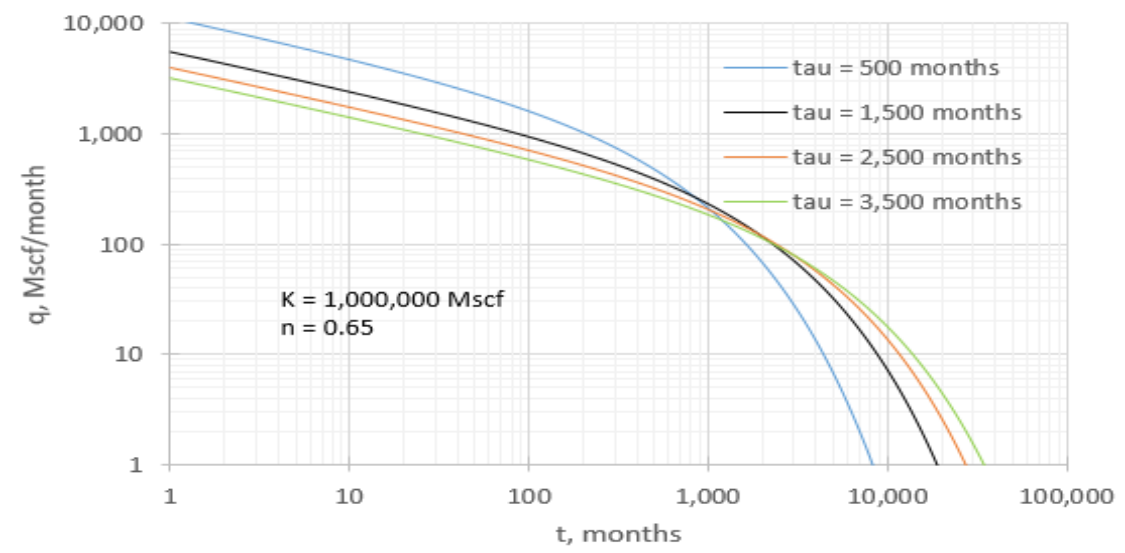

Figure 9: A logarithmic plot of production rate against time. The plot is based on Equation 15. Small values of tau yield relatively high rates at early times, while, at late times, large values of tau yield relatively high rates.

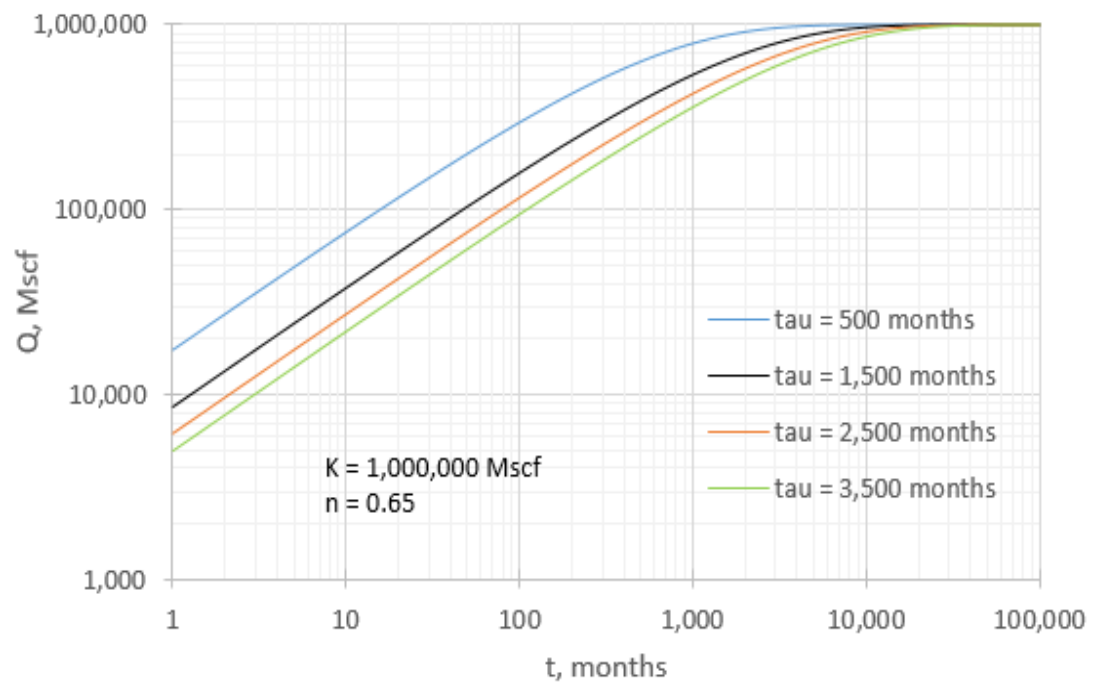

Figure 10: A logarithmic plot of cumulative production against time. The plot is based on Equation 14. Small values of tau yield relatively high values for a prolonged period. At large times, however, the curves converge and approach the value of the carrying capacity.

\subsubsection{Effect of the carrying capacity}

Figures 11 and 12 show the effect of the carrying capacity on the production performance of a fractured-well in unconventional reservoir. Throughout the production period, higher values of the carrying capacity yield higher rates and cumulative production. Because the carrying capacity reflects the volume of fluid in-place, it is no surprise that large amount of fluid will be produced from a reservoir with a high carrying capacity, and vice versa. 


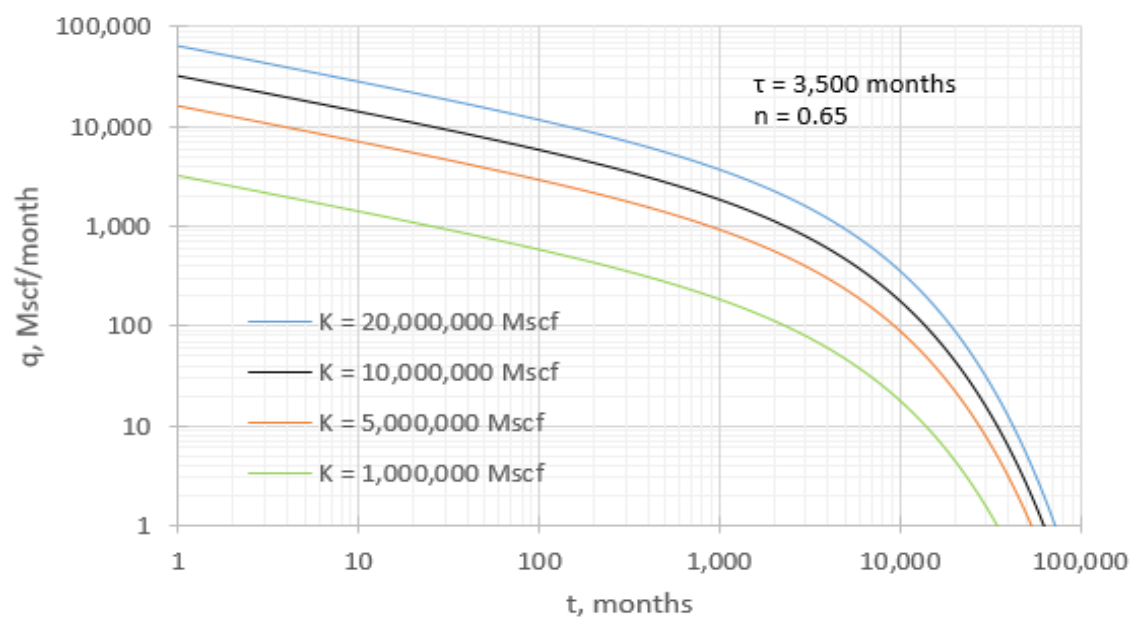

Figure 11: A logarithmic plot of production rate against time. The plot is based on Equation 14. Clearly, the higher the carrying capacity, the higher the production rate.

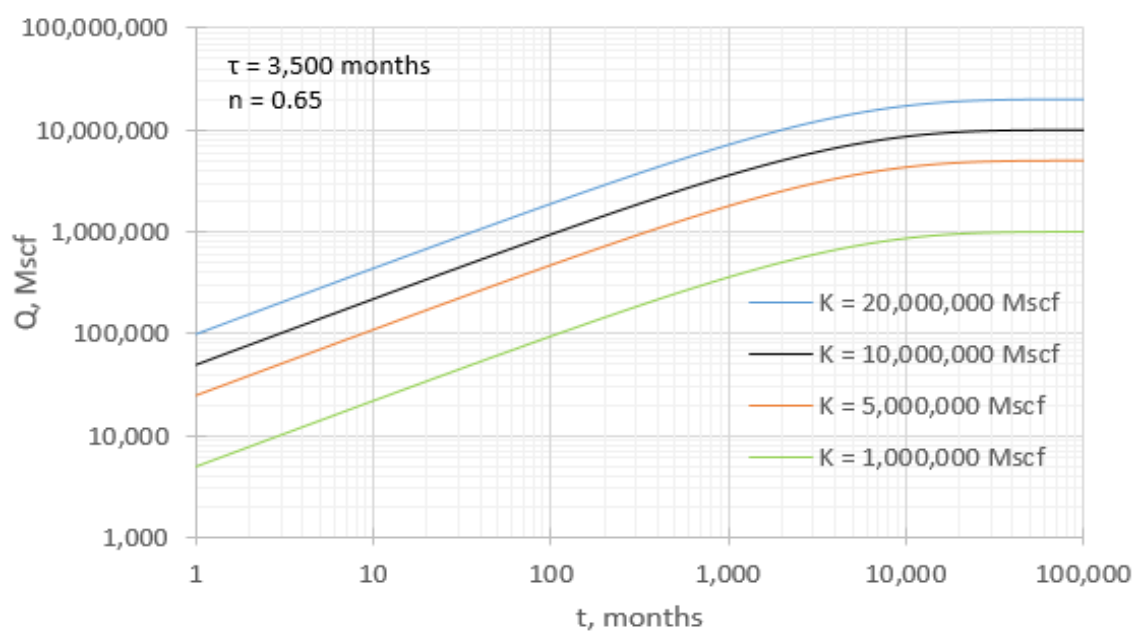

Figure 12: A logarithmic plot of cumulative production against time. The plot is based on Equation 15. Clearly, the higher the carrying capacity, the higher the cumulative production.

\subsubsection{Effect of the carrying capacity on $z(K, Q)$}

Figure 13 shows the sensitivity of $\mathrm{z}(\mathrm{K}, \mathrm{Q})$ to the carrying capacity, $\mathrm{K}$. The data is from Barnett shale (Browning et al. 2013). Underestimated and overestimated $\mathrm{K}$ values cause the data points to bend upwards and downwards at late times, respectively. The correct K value, however, linearizes the entire data points. Notice that, at early times, the data points are linearized regardless of the K value used. Therefore, it is recommended that the $\mathrm{K}$ value is determined with well-known methods, such as the volumetric method, if possible. 


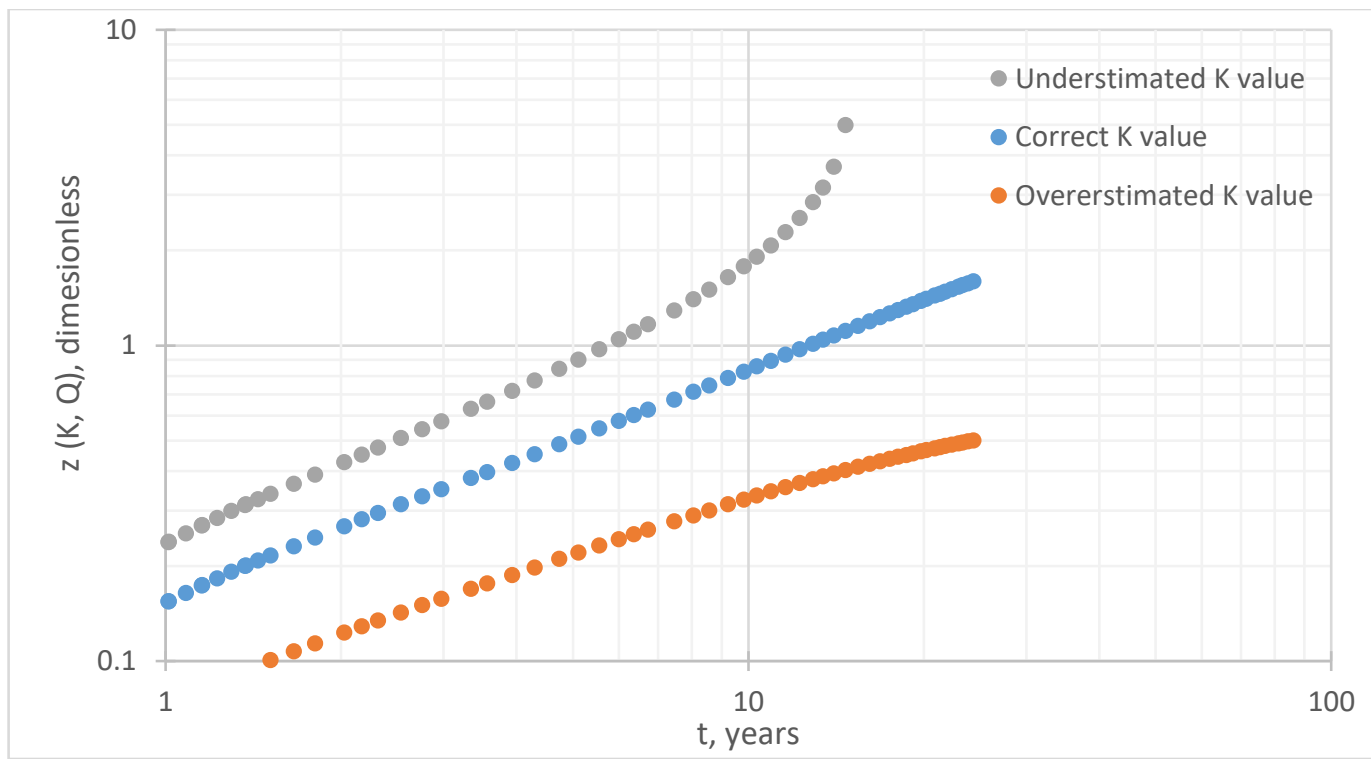

Figure 13: Sensitivity of $\mathrm{z}(\mathrm{K}, \mathrm{Q})$ to the technically recoverable resource, K. Underestimated and overestimated $\mathrm{K}$ values cause the data points to bend upwards and downwards at late times, respectively. The data points are, however, linearize when the correct $\mathrm{K}$ value is used.

\subsection{Applications}

Having validated the PLSE model, and explored the sensitivities of the model parameters, here, we apply the PLSE model to field data. Also, a comparison with the existing models is made.

\subsubsection{Case 2: North Dakota Well B}

The field data in this case is from a fractured gas well in a low-permeability reservoir, which is located in North Dakota, New Mexico. The production data from Well B is presented in Figure 14. Note that the data points after 5000 days appear not to follow the general trend; this may be due to fracture interference (Gupta et al. 2019) or a newly drilled nearby well (Al Alhmadi and Wattenbarger 2011). Based on volumetric method, the carrying capacity, K, is 780,000 Mscf. In Figure 15, a logarithmic plot of $\mathrm{z}(\mathrm{K}, \mathrm{Q})$ against time is constructed. The data is linearized, and the model parameters ( $n$ and $\tau$ ) are estimated from the slope and intercept. The Duong's model has been included. The PLSE model performs better at linearizing the data. This is, in part, due to the cumulative production being used in $\mathrm{z}(\mathrm{K}, \mathrm{Q})$ as opposed to the flow rate, which is noisy. The model parameters are presented in Table 2. Model parameters for Arps' model and the LGM have also been presented in Table 2 .

Figures 16 and 17 show the history matching and production prediction performance of the various models on a log-log and semi-log graphs, respectively. The Duong's model and the LGM show a slight incline phase at early times. Furthermore, while the Duong's model yields higher prediction at late time, the LGM underestimates the production at late times. The PLSE and the Arps' models seem to trace the production data quite well. It is apparent in Figure 17 (the semilog graph) that the Duong's model and LGM exhibit an "artificial" incline phase, which is not a behaviour of the data being analyzed. Figure 18 shows the cumulative production plot. Clearly, the Duong's model and the LGM overestimates and underestimates the cumulative production, 
respectively. On the other hand, although the Arps' model and the PLSE model perform quite well, the PLSE model seems to be more conservative than the Arps' model.

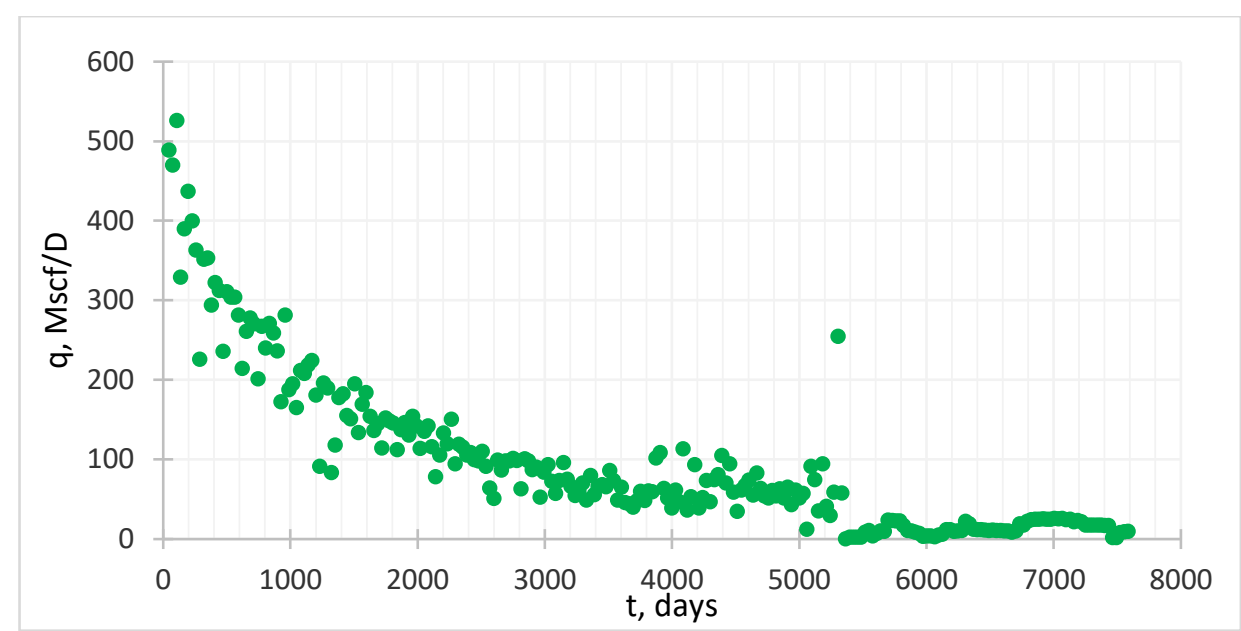

Figure 14: Rate history of a fractured gas well Dakota field for case 2.

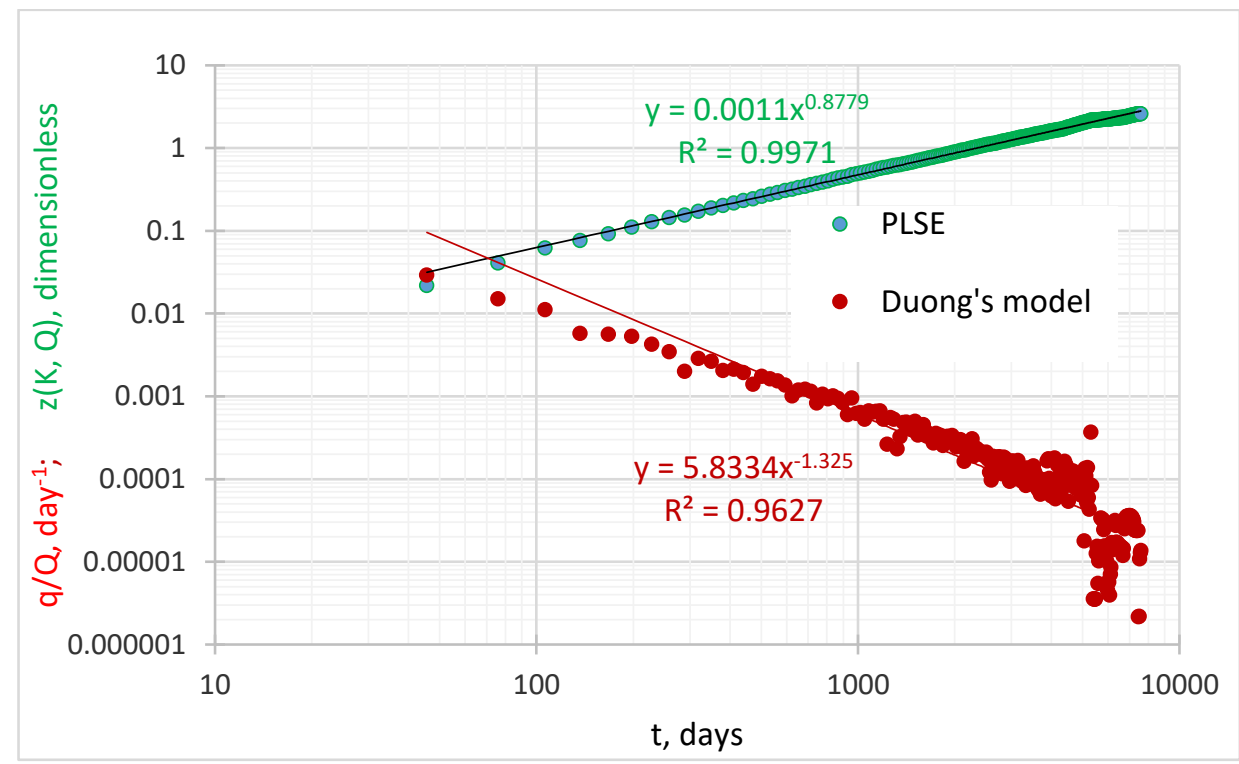

Figure 15: A log-log graph of the PLSE model and the Duong's model. Because the PLSE model circumvents the use of flow rate, it linearizes the data points better than the Duong's model. 


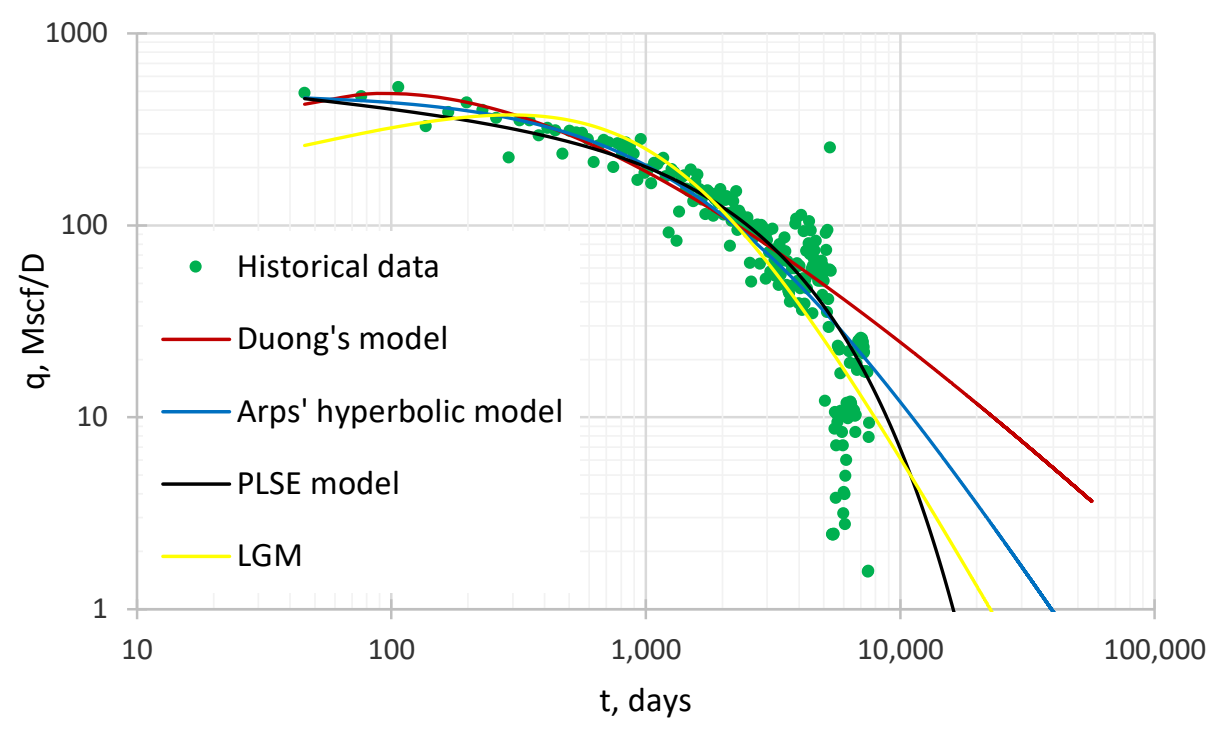

Figure 16: Production performance comparison of the PLSE model and other existing models on a logarithmic graph. The PLSE model seems to be the most conservative. The data points that deviate from the trend are outliers, may be due to fracture interference or fracture hit.

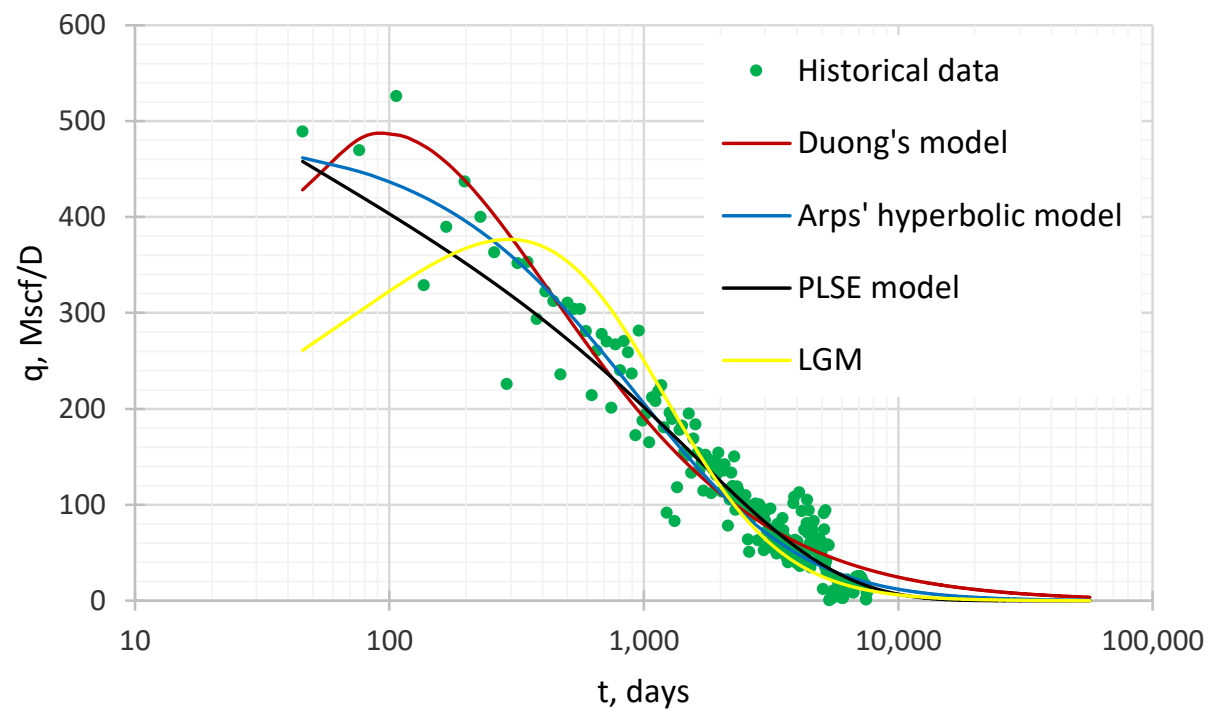

Figure 17: Production performance comparison of the PLSE model and other existing models on a semi-log graph. It is apparent in this graph that the Duong' model and LGM exhibit an "artificial" incline phase, which is not a behaviour of the data being analyzed. 


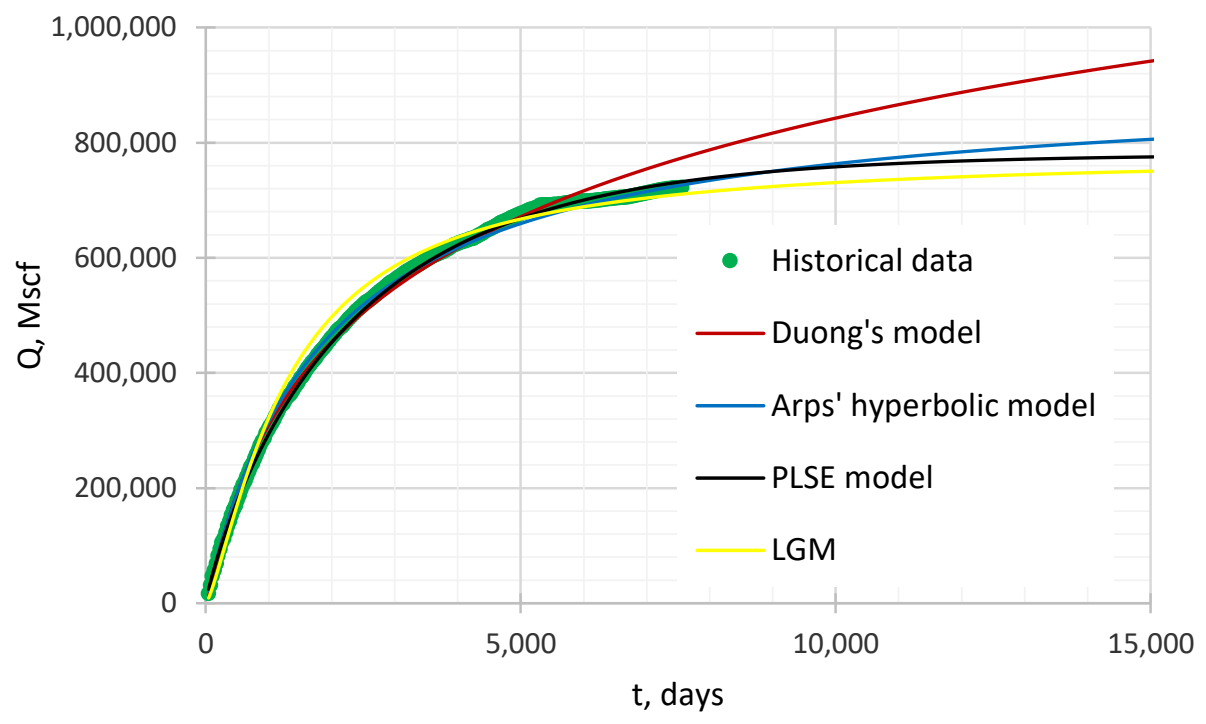

Figure 18: Cumulative production performance comparison of the PLSE model and other existing models on a linear graph. While the Duong' model and the LGM fail to match the data at late times, the Arps model yields higher prediction than the PLSE model.

Table 2: Estimated model parameters for case 2

\begin{tabular}{|c|c|c|c|c|c|c|c|c|c|c|c|}
\hline \multicolumn{3}{|c|}{ Duong's model } & \multicolumn{3}{|c|}{ Arps' model } & \multicolumn{3}{|c|}{ PLSE } & \multicolumn{3}{|c|}{ LGM } \\
\hline $\mathrm{q} 1$ & $\mathrm{a}$ & $\mathrm{m}$ & qi & Di & $\mathrm{b}$ & $\mathrm{K}$ & $\tau$ & $\mathrm{n}$ & $\mathrm{K}$ & $\mathrm{a}$ & $\mathrm{n}$ \\
\hline 0.1937 & 5.8334 & 1.325 & 484 & 0.00107 & 0.5 & 780000 & 2245 & 0.8779 & 780000 & 13326 & 1.324 \\
\hline
\end{tabular}

\subsubsection{Case 3: Anderson County, Texas}

Case 3 is an oil-well data, which span from January 1993 to January 2020. The data was obtained from the public domain (Railroad Commission, Texas). The production data is presented in Figure 19. In this case, as with public data, the rock and fluid parameters were not available, and so nonlinear regression was used. In particular, Excel Solver was used to estimate the parameters. Having determined the carrying capacity, $\mathrm{K}$, from the Solver, Equation 18 was used to validate the estimates. The plot is shown in Figure 20. The data is linearized, and the model parameters ( $\mathrm{n}$ and $\tau)$ are estimated from the slope and intercept. The Duong's model has been included. The model parameters are presented in Table 3. Model parameters for Arps' and LGM have also been presented.

Figures 21 and 22 show the history matching and production prediction performance of the various models on a log-log and semi-log graphs, respectively. Again, the Duong's model yields higher prediction at late time, and the LGM underestimates the production at late times. Although the PLSE and the Arps' models seem to trace both the transient-state boundary-dominated flow periods quite well, the PLSE model is more conservative. Figure 23 shows the cumulative production plot. Clearly, the Duong's model and the LGM overestimates and underestimates the cumulative production, respectively. On the other hand the Arps' model and the PLSE model perform quite well. 


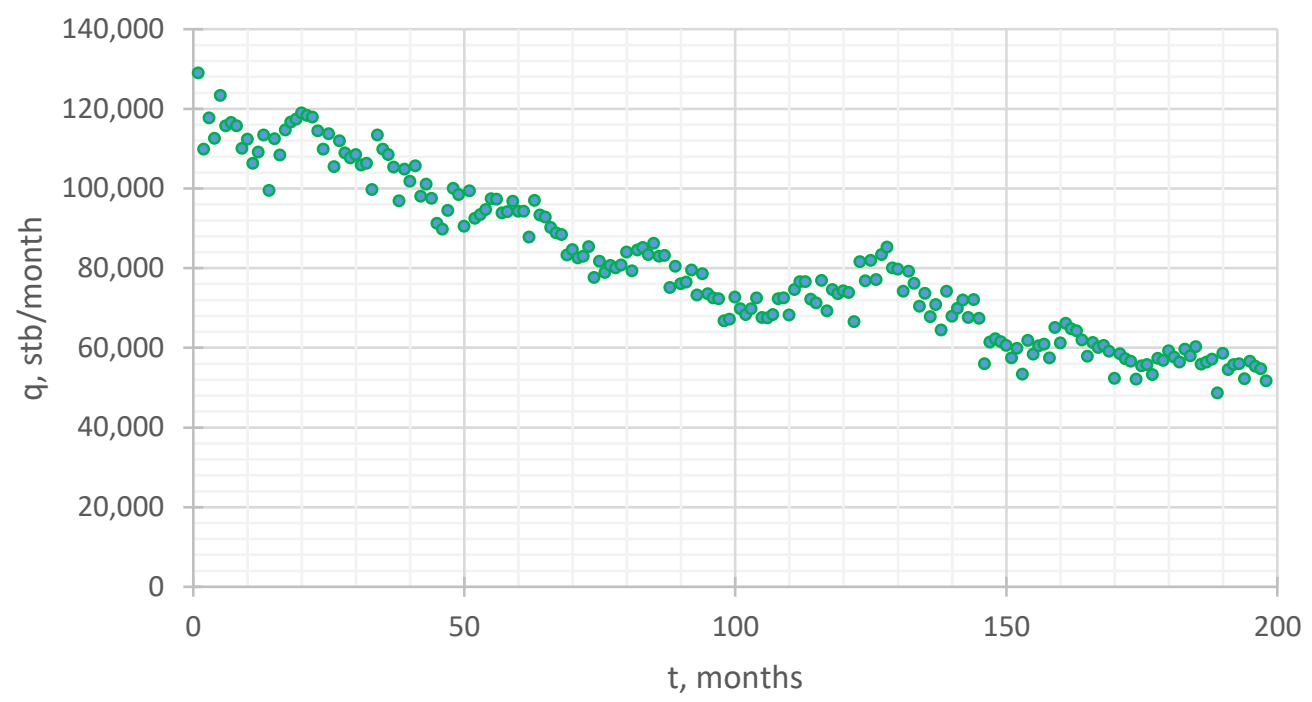

Figure 19: Rate history of a fractured oil well for case 3.

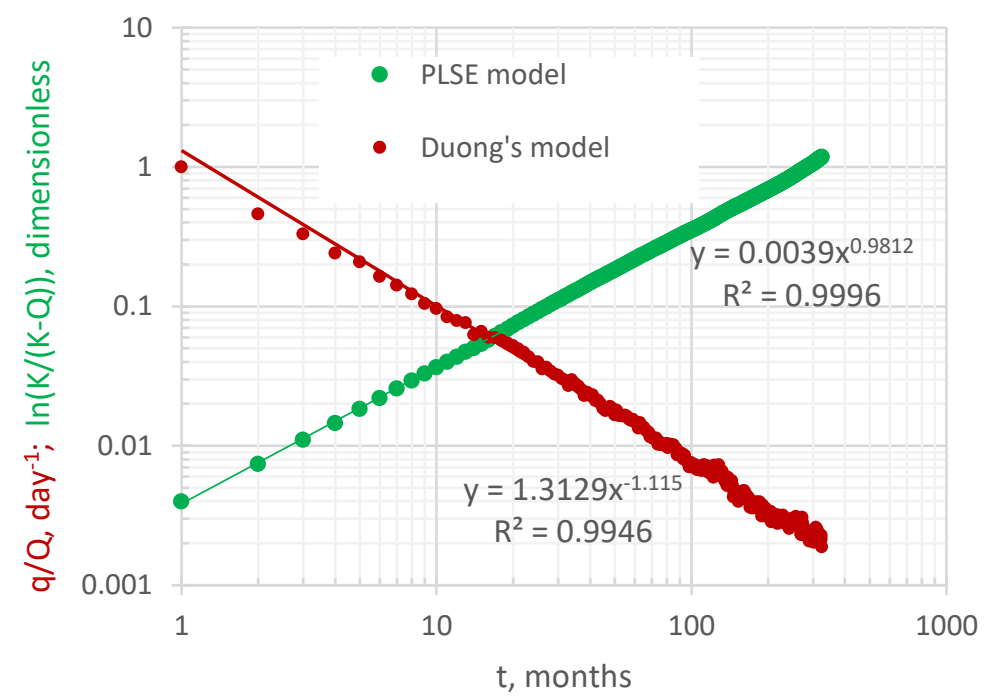

Figure 20: A log-log graph of the PLSE model and the Duong's model for case 3. 


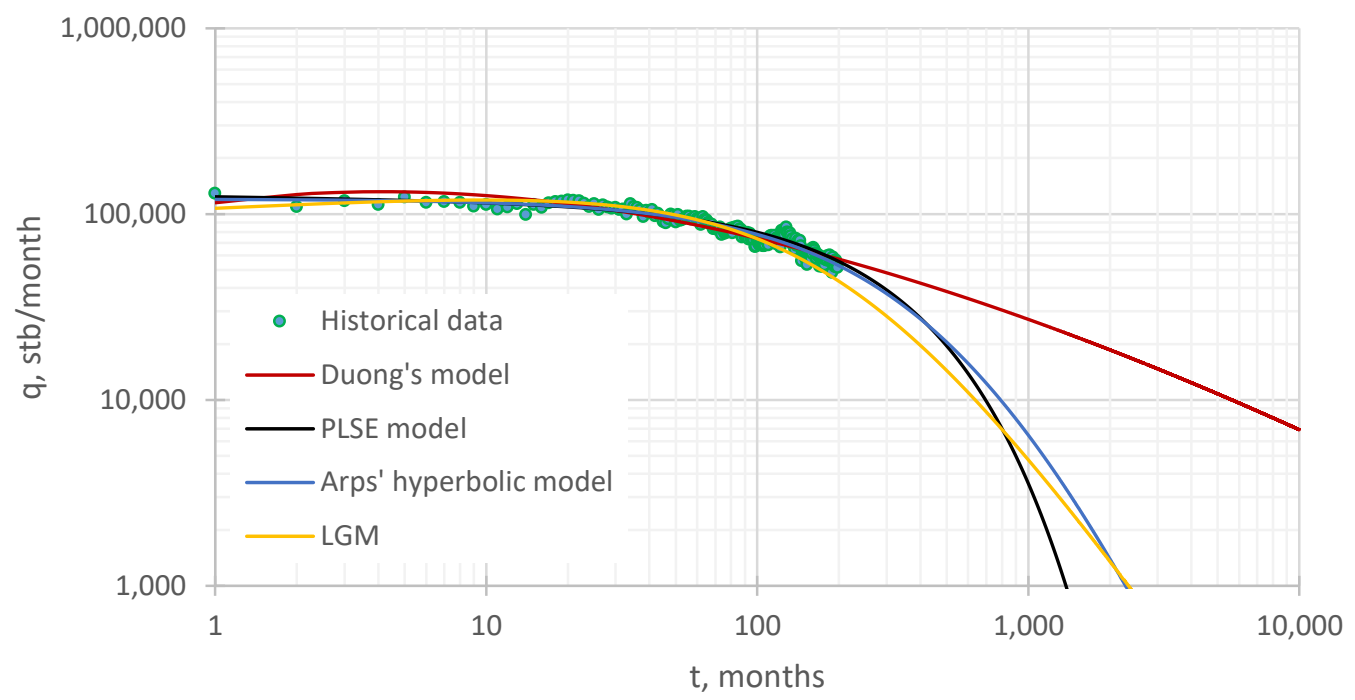

Figure 21: Production performance comparison of the PLSE model and other existing models on a logarithmic graph for case 3 . The PLSE model seems to be the most conservative.

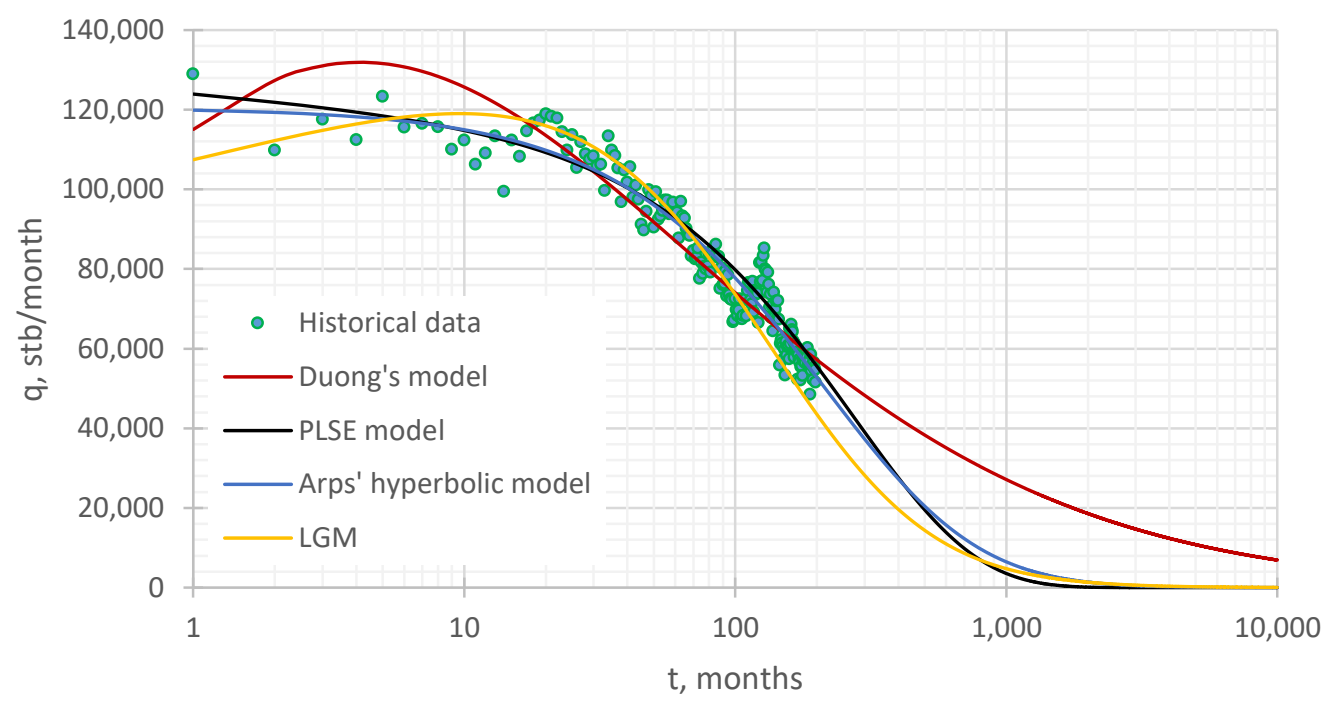

Figure 22: Production performance comparison of the PLSE model and other existing models on a semi-log graph. At both early- and late times, the Arps' hyperbolic model and PLSE model give good estimates. The Duong' model overestimate the production prediction at late times, and also exhibit, at early times, an incline phase, which is uncharacteristic of the data being analyzed. 


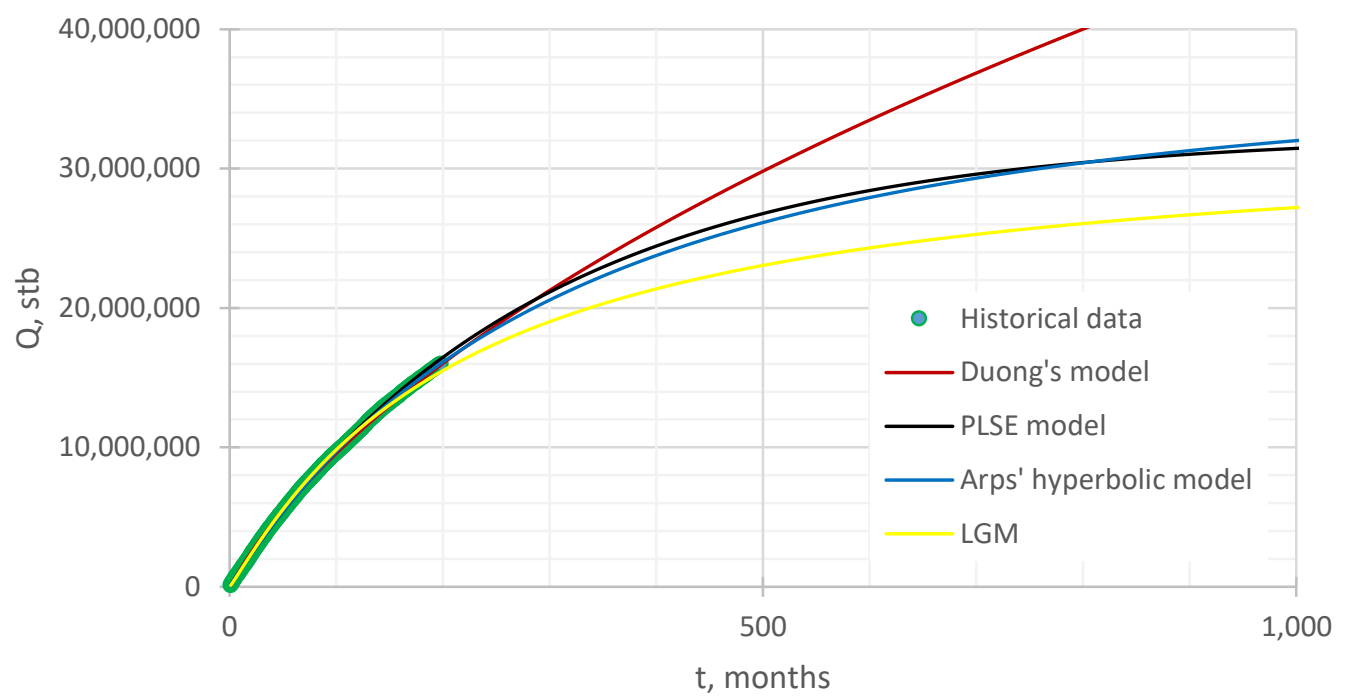

Figure 23: Cumulative production performance comparison of the PLSE model and other existing models on a linear graph. While the Duong' model and the LGM fail to match the data at late times, the PLSE model and the Arps' model yield good estimate.

Table 3: Estimated model parameters for case 3

\begin{tabular}{|c|c|c|c|c|c|c|c|c|c|c|c|}
\hline \multicolumn{3}{|c|}{ Duong's model } & \multicolumn{3}{|c|}{ Arps' model } & \multicolumn{3}{|c|}{ PLSE } & \multicolumn{3}{|c|}{ LGM } \\
\hline $\mathrm{q} 1$ & $\mathrm{a}$ & $\mathrm{m}$ & qi & Di & $\mathrm{b}$ & $\mathrm{K}$ & $\tau$ & $\mathrm{n}$ & K & $\mathrm{A}$ & $\mathrm{n}$ \\
\hline 0.1937 & 1.3129 & 1.115 & 120432 & 0.00468 & 0.3 & $32,507,377$ & 285 & 0.9812 & $32,507,377$ & 323 & 1.073 \\
\hline
\end{tabular}

\subsubsection{Case 4: Baylor County, Texas}

Case 4 is an oil-well data, which span from January 1993 to April 2020. The data was obtained from the public domain (Railroad Commission, Texas). The production data is presented in Figure 24. Again, having determined the carrying capacity, K, from the Excel Solver, Equation 18 was used to validate the estimates. The plot is shown in Figure 25. The data is linearized, and the model parameters ( $\mathrm{n}$ and $\tau)$ are estimated from the slope and intercept. The model parameters are presented in Table 4.

Figures 26 and 27 show the history matching and production prediction performance of the various models on a log-log and semi-log graphs, respectively. In this case, the Arps' hyperbolic model and the Duong's model yield higher prediction at late time, and the LGM underestimates the production at late times. The PLSE model traces both the transient-state and boundarydominated flow periods quite well. Figure 28 shows the cumulative production plot. Clearly, the Arps' hyperbolic model and the Duong's model overestimate the cumulative production, while he PLSE and the LGM yield good estimates at early and late times. 


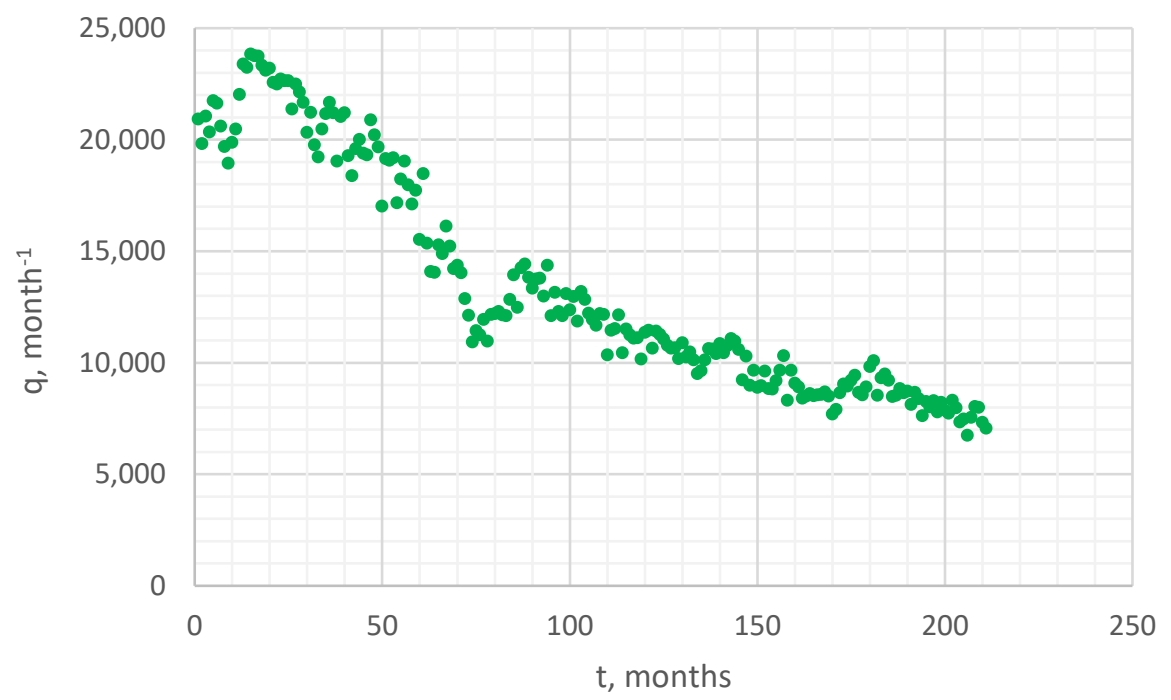

Figure 24: Rate history of a fractured oil well for case 4.

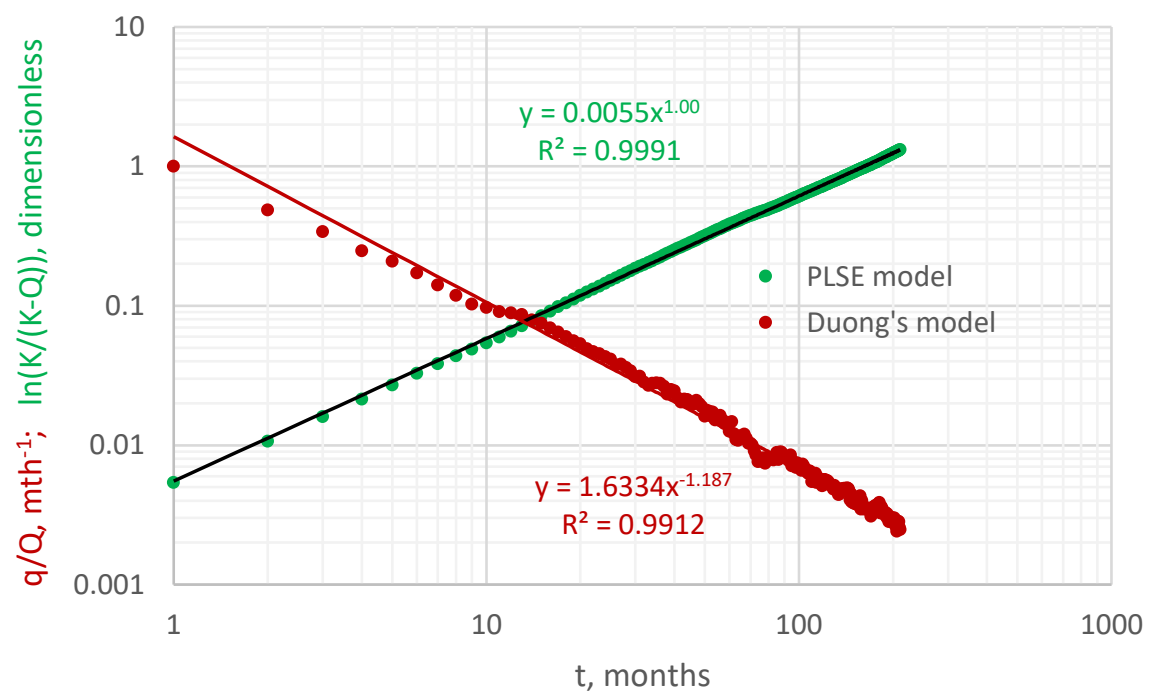

Figure 25: A log-log graph of the PLSE model and the Duong's model for case 4. 


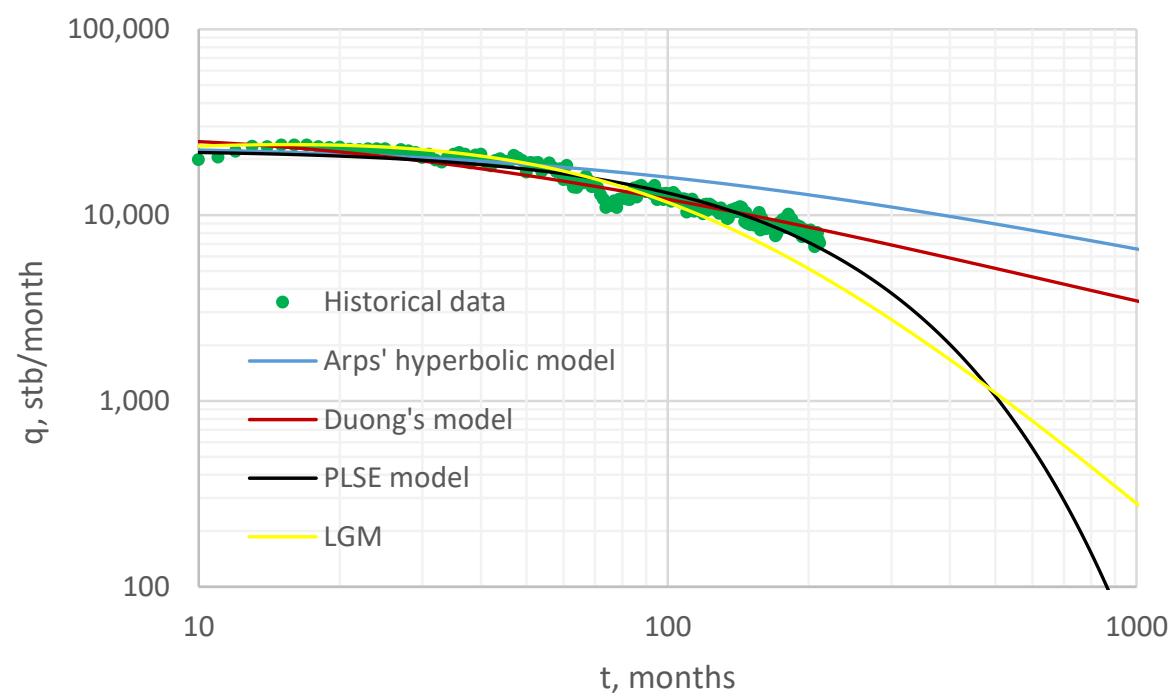

Figure 26: Production performance comparison of the PLSE model and other existing models on a logarithmic graph for case 4 . The PLSE model to be the most conservative.

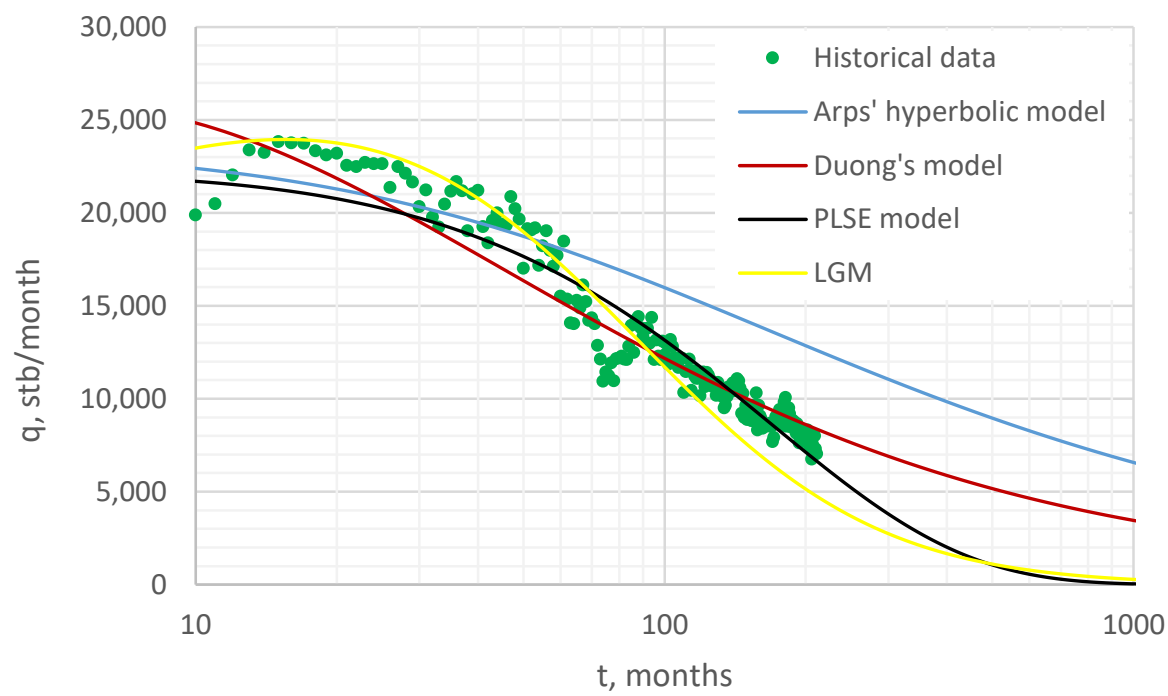

Figure 27: Production performance comparison of the PLSE model and other existing models on a semi-log graph for case 4. The Arps' hyperbolic model gives higher rate predictions at late times, and LGM underestimates the production rate at late times, though it performs well at early time. The PLSE model give good estimates at late times, but no so good at early time. Nevertheless, overall, the PLSE seems to give the best prediction among all the models. 


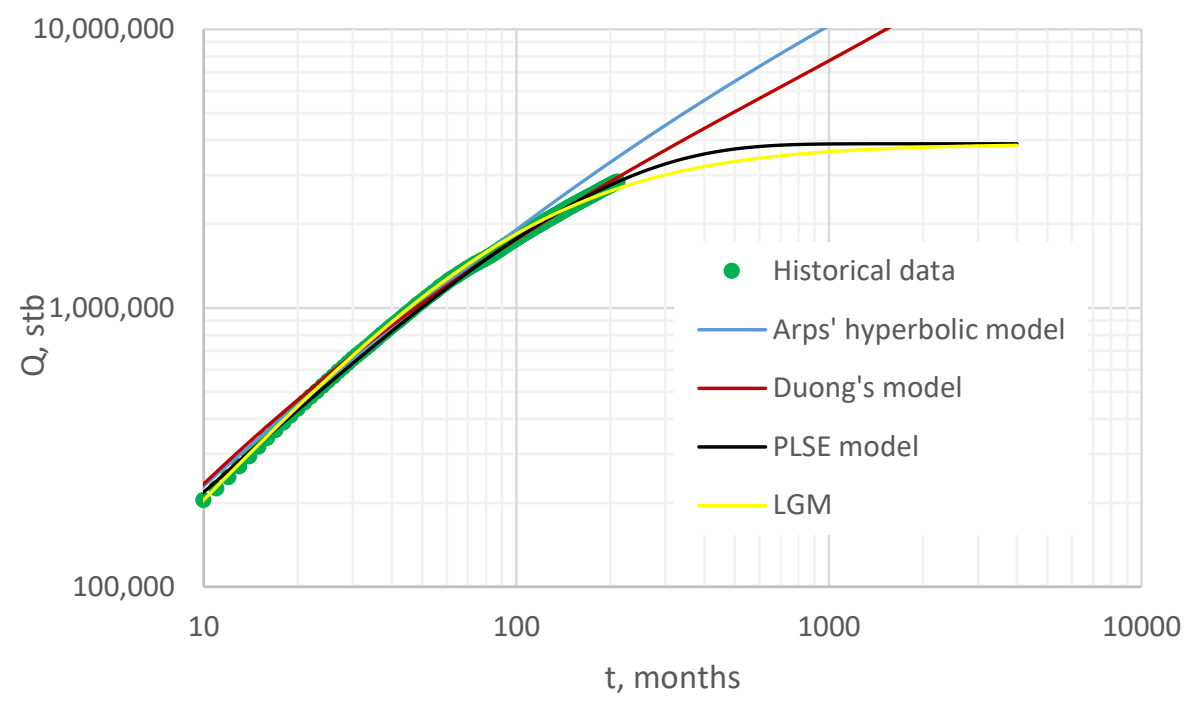

Figure 28: Cumulative production performance comparison of the PLSE model and other existing models on a linear graph for case 4. The Duong' model and the Arps' model overestimate the data at late times, the LGM slightly underestimates the data at late time. The PLSE model, on the other hand, gives good predictions both at early and late times.

Table 4: Estimated model parameters for case 4

\begin{tabular}{|c|c|c|c|c|c|c|c|c|c|c|c|}
\hline \multicolumn{3}{|c|}{ Duong's model } & \multicolumn{3}{|c|}{ Arps' model } & \multicolumn{3}{|c|}{ PLSE } & \multicolumn{3}{|c|}{ LGM } \\
\hline $\mathrm{q} 1$ & $\mathrm{a}$ & $\mathrm{m}$ & qi & Di & $\mathrm{b}$ & $\mathrm{K}$ & $\tau$ & $\mathrm{n}$ & $\mathrm{K}$ & $\mathrm{a}$ & $\mathrm{n}$ \\
\hline 17440 & 1.6334 & 1.187 & 23700 & 0.006 & 2 & $3,881,791$ & 162 & 1.00 & $3,881,791$ & 291 & 1.210 \\
\hline
\end{tabular}

\subsubsection{P10, P50 and P90 type curves for the Barnett shale}

Groups of wells, called Tiers, from the Barnett shale are analyzed to estimate P10, P50 and P90 values, and to develop P10, P50 and P90 type curves for the Barnett shale. The production data was previously presented by Browning et al. (2013). Figure 29 shows the production history for each of the tiers (or groups of wells). Tiers 1 through 10 have the highest to the lowest production profile, respectively.

The production data of each of the tiers has been analyzed with the proposed PLSE model. Figure 30 shows the cumulative probability of exceedance as a function of the carrying capacity. The plot assumes an exponential decline fit. The data is re-plotted on a linear graph as shown in Figure 31, and fitted with an exponential model as it is easier to read from a linear graph. The P10, P50 and P90 estimates of K are extracted from Figure 31. Similar analysis has been used to estimate the $P 10, P 50$ and P90 values for $n$ and $\tau$. Figures 32 and 33 show the cumulative probability for $n$ and $\tau$ cases, respectively. The P10, P50 and P90 estimates of the model parameters are presented in Table 5.

As shown in Figures 34 (linear graph) and 35 (log-log graph), the P10, P50 and P90 rate profiles have been superposed on the production prolife of the Tiers. As expected, the P10 curve, which is optimistic, lies above the production profile of the Tiers curves, except Tier 1; the P90 curve, which is the most conservative, lies below the Tiers curves, except Tiers 1 and 2; and the P50 
curve, which is somewhat the average, lies somewhat in the middle of the Tiers curves. Thus, depending on the objective, the appropriate P10, or P50 or P90 curve can be used to forecast the Barnett shale. The P10, P50 and P90 cumulative production profiles are shown in Figure 36. The P10 curve yields optimistic estimates; the P90 yields pessimistic estimates; and the P50 curve yields average estimates.

Figure 37 shows a composite plot of the P10, P50 and P90 type curves for rate and cumulative production. The type curves have been developed by normalizing the rate and cumulative production by the K10 value (the P10 estimate of the carrying capacity). The plotting function on the $\mathrm{X}$-axis is in real times, which is advantageous because it allows predictions in real time. The P10, P50 and P90 type curves may be useful for predicting the production performance of analogue wells.

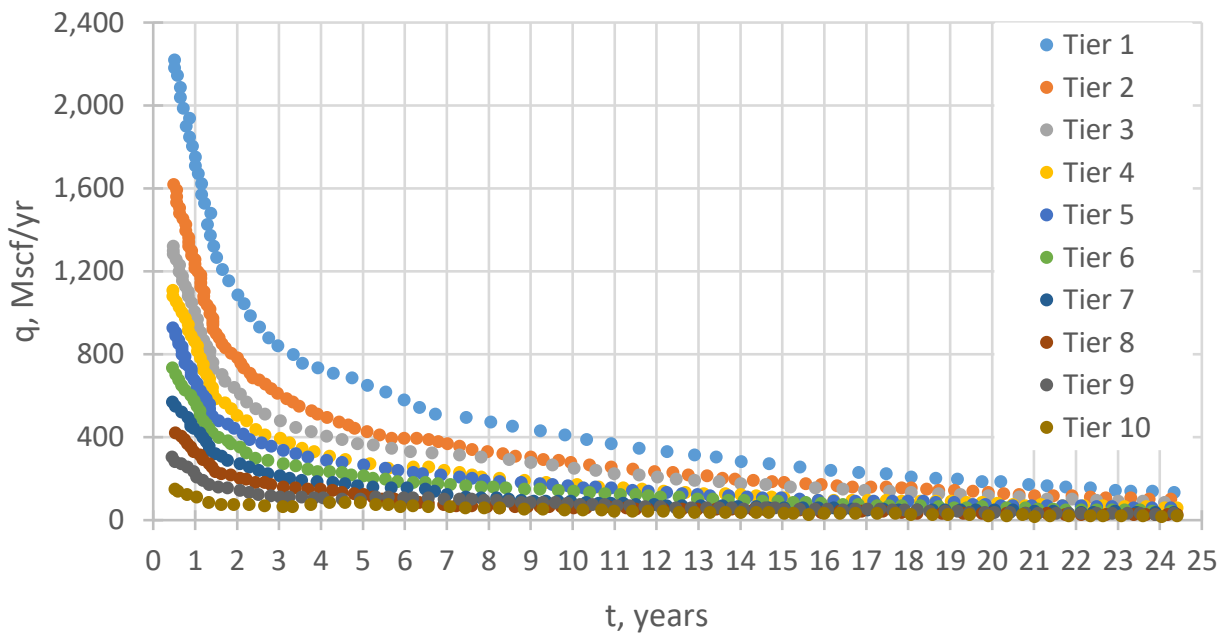

Figure 29: Production history of the tiers for the Barnett shale. The data has been taken from Browning et al. (2013).

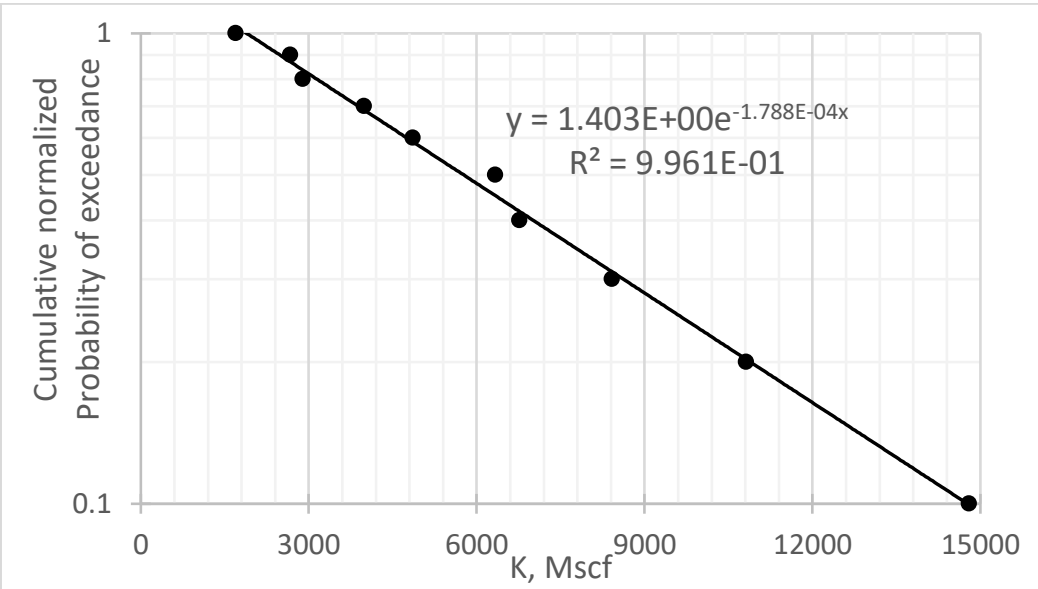

Figure 30: A semi-log plot of cumulative normalized probability of exceedance of the tiers for the Barnett shale. The straight-line suggests that the plot follows an exponential fit. 


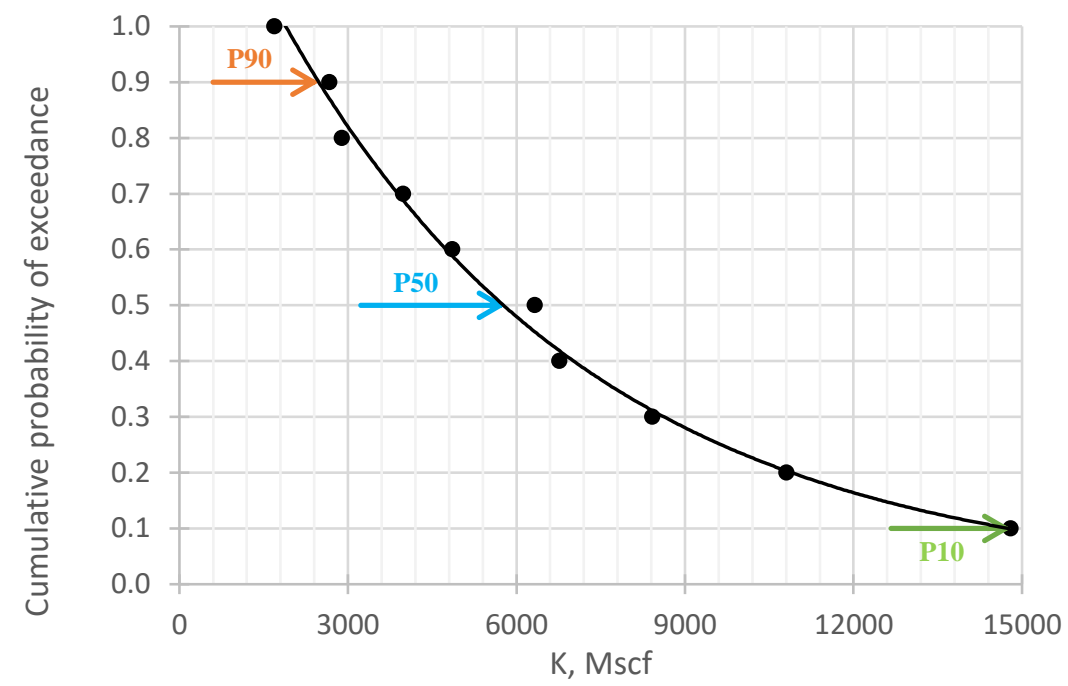

Figure 31: A linear plot of cumulative normalized probability of exceedance of the tiers for the Barnett shale. The plot allows the P10, P50 and P90 estimates of K.

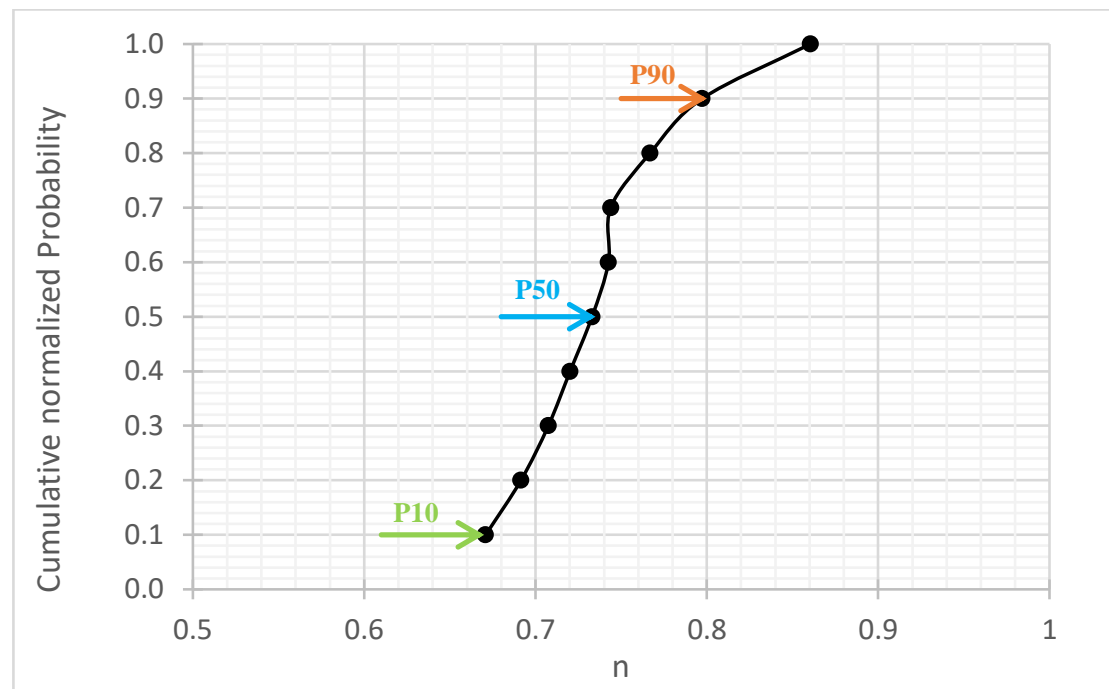

Figure 32: A linear plot of cumulative normalized probability against the decline exponent of the tiers for the Barnett shale. The plot allows the P10, P50 and P90 estimates of $\mathrm{n}$. 


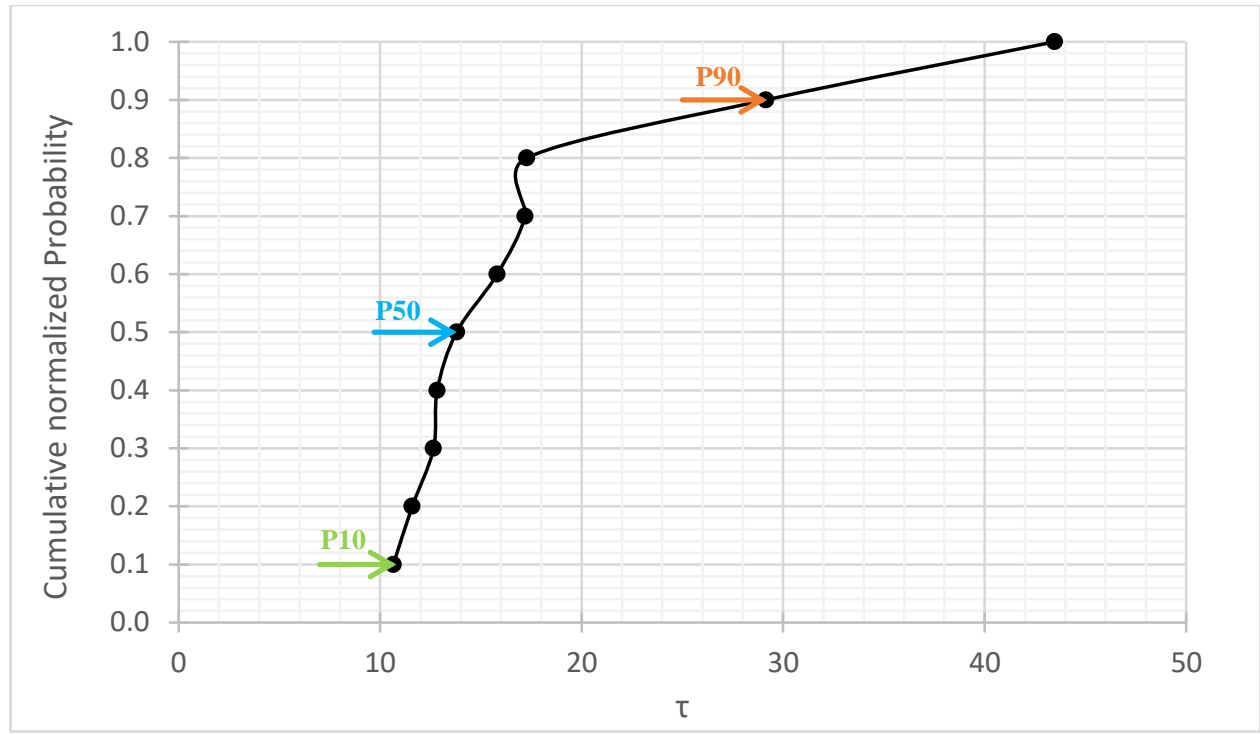

Figure 33: A linear plot of cumulative normalized probability against the characteristic time of the tiers for the Barnett shale. The plot allows the P10, P50 and P90 estimates of $\tau$.

Table 5: P90, P50 and P10 estimates of the model parameters for the Barnett shale

\begin{tabular}{|c|c|c|c|c|c|c|c|c|}
\hline \multicolumn{3}{|c|}{ K, Mscf } & \multicolumn{3}{|c|}{$\mathrm{n}$, dimensionless } & \multicolumn{3}{|c|}{$\tau$, years } \\
\hline P90 & P50 & P10 & P90 & P50 & P10 & P90 & P50 & P10 \\
\hline 2,480 & 5,800 & 14,797 & 0.80 & 0.73 & 0.67 & 29.2 & 13.8 & 10.8 \\
\hline
\end{tabular}

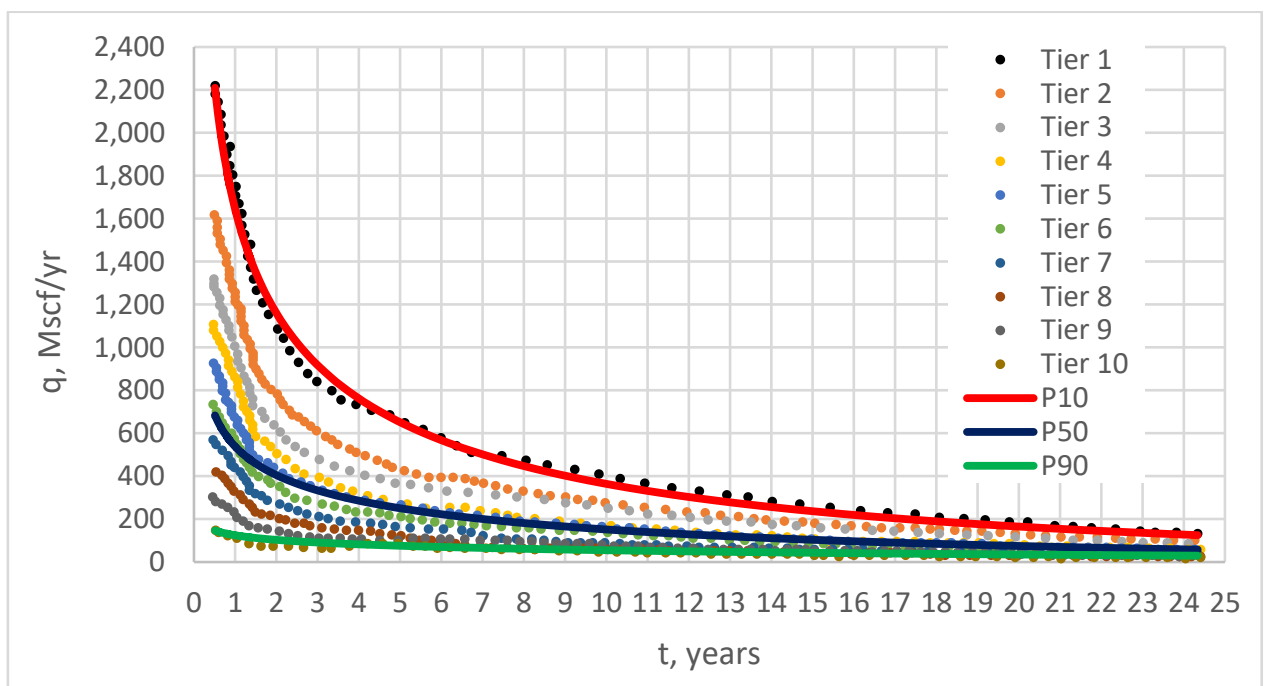

Figure 34: A liner plot of the flow rate history of the tiers with the P10, P50 and P90 production profiles superposed on them for the Barnett shale. As expected, the P90 curve gives the most conservative estimates. 


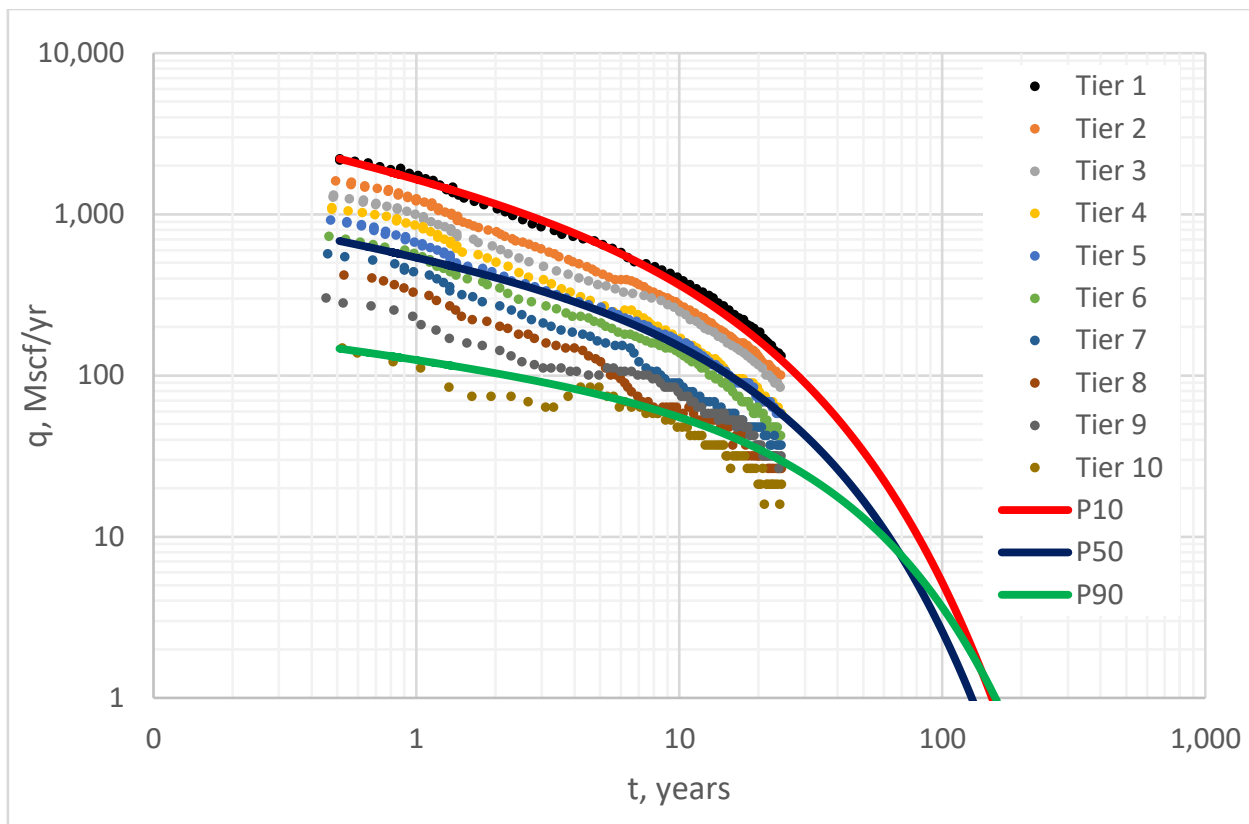

Figure 35: A log-log plot of the flow rate history of the tiers with the P10, P50 and P90 production profiles superposed on them for the Barnett shale. The P90 curve gives the most conservative estimates.

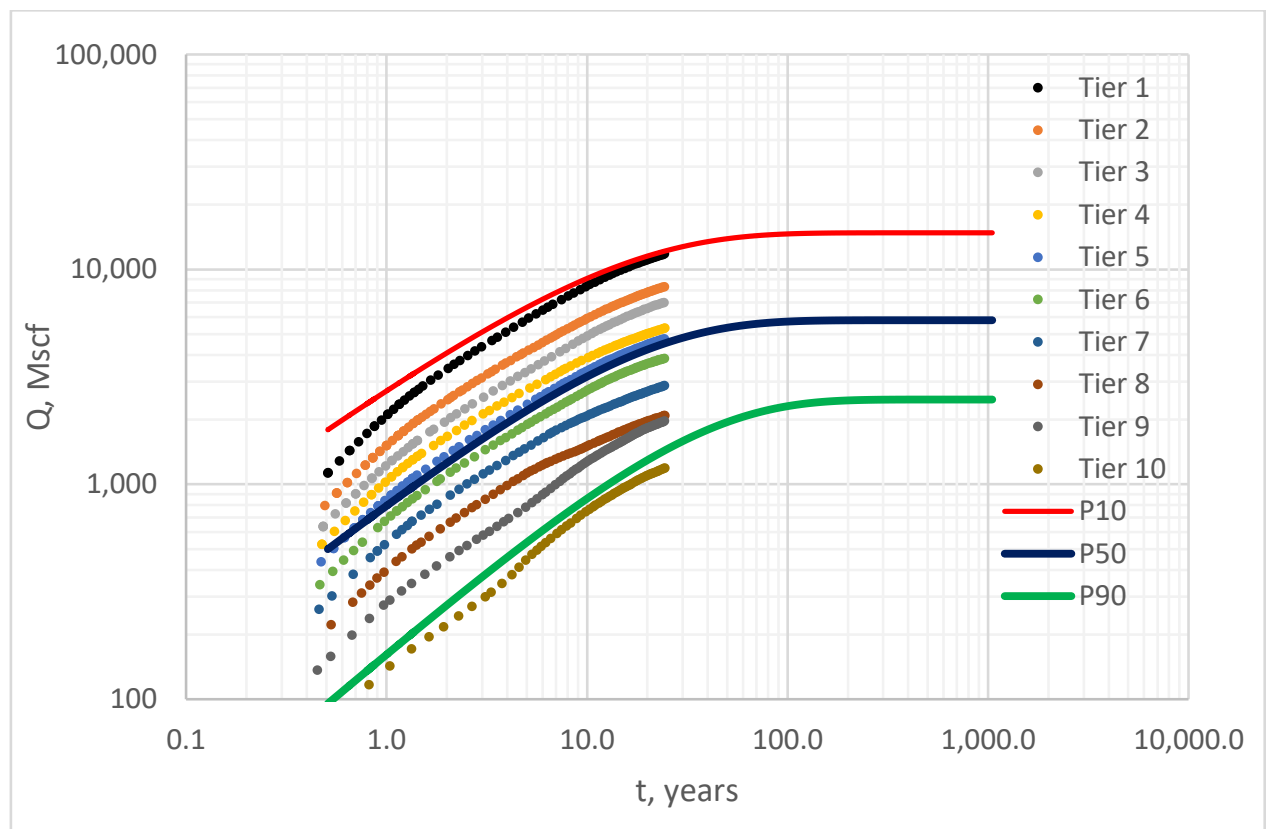

Figure 36: A log-log plot of the cumulative production profile of the tiers with the P10, P50 and P90 cumulative profiles superposed on them for the Barnett shale. The P90 curve gives the most conservative estimates. 


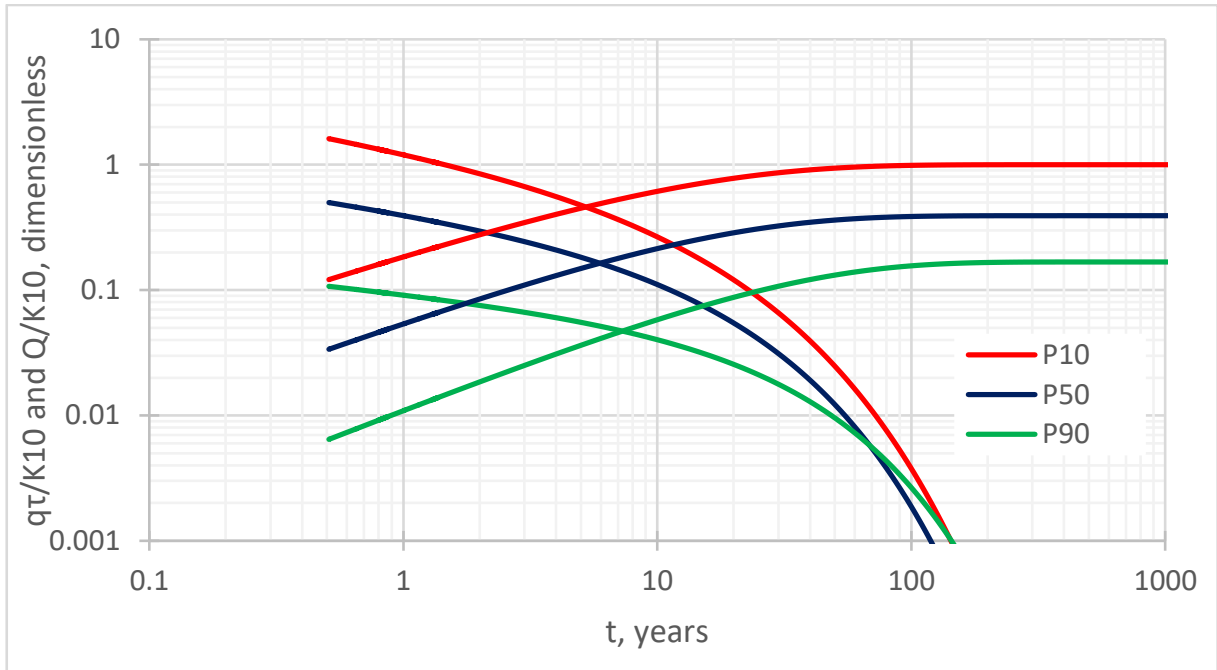

Figure 37: P10, P50 and P90 rate and cumulative production type curves for the Barnett shale. The P90 curve gives the most conservative estimates. These types curve may be useful for analyzing analogue wells.

\subsection{Conclusions}

The primary objective of this paper has been to propose a production-based model to analyze and forecast a fractured-well or a cluster of wells performance in unconventional reservoirs. The model has been derived based on a modified version of the Duong's model. In particular, a carrying capacity has been incorporated into the Duong's model to eliminate any artificial incline phase and impose a bound at late times. The model has been applied to several field data, and the results have been compared with existing models. Based on the findings, the following conclusions have been drawn:

1. The proposed production-based model is a power-law function with a stretched exponential cut-off; numerical and field data have suggested that the proposed power-law stretched exponential (PLSE) model trace the production data both at early-time and late-time periods;

2. At very early times, the Duong's model gives an artificial incline phase; also, the Duong's model overestimates production prediction during boundary-dominated flow period; however, the Duong's model is well suited during the transient-state period;

3. The Arps' model traces the boundary-dominated flow data when $b<1$; it traces the transient state if $b>1$; however, there is no particular value of $b$ that allows the Arps' model to trace the transient-state and boundary-dominated flow data simultaneously;

4. The logistic growth model is well suited during the transient state period; unfortunately, it underestimates production during the boundary-dominated flow period; also, similar to the Duong's model, the LGM model gives an artificial incline phase at very early times;

5. Compared with the existing models - Duong's model, Arps' hyperbolic model and logistic growth model - the PLSE model seems to be consistent in tracing both the transient-state and boundary-dominated flow data, and yields relatively good estimates; 
6. P10, P50 and P90 rate and cumulative type curves for the Barnett shale have been developed using the proposed PLSE model. These type curves may be useful for analyzing and predicting analogue wells' future production performance of the Barnett shale.

\section{Acknowledgements}

This study was carried out at the University of Energy and Natural Resources, Sunyani, Ghana. The authors acknowledge the computing facility provided by the University.

\section{References}

Al- Ahmadi, A. H, and Wattenbarger, R. A. 2011. Triple-porosity models: One further step towards capturing fractured reservoirs heterogeneity. SPE/DGS Saudi Arabia Section Technical Symposium and Exhibition. Al-Khobar: Society of Petroleum Engineers. 1-21.

Al-Ahmadi, H. A., Almarzooq, A. M. and Wattenbarger, R. A. 2010. Application of linear flow analysis to shale gas wells - Field cases. Paper SPE 130370 presented at the 2010 Unocnetional Gas Conference. Pittsburgh, Pennsylvania: Society of Petroleum Engineers.

Arps, J. J. 1945. Analysis of Decline Curves. SPE-945228-G. Trans., AIMS 160: 228-247.

Barraud, C., Atteia, J, L., Olive, J. F. et al. 2002. First results of Hete-2/Fregate. Proceedings, $37^{\text {th }}$ Rencontres de Moriond, The gamma-ray universe. 497-500.

Bello, R. O. and Wattenbarger, R. A. 2010. Multistage hydraulically fractured shale gas rate transient analysis. Paper SPE-126754 presented at the North Africa Technical Conference and Exhibition. Cairo, Egpyt: Society of Petroleum Engineers.

Bello, R. O. and Wattenbarger, R. A. 2008. Rate transient analysis in naturally fractured shale gas reservoirs. Paper SPE 114591 presented at the CIPC/SPE Gas Technology Symposium 2008 Joint Conference. Calgary, Alberta: Society of Petroleum Engineers.

Bello, R. O. 2009. Rate transient analysis in shale gas reservoirs with transient linear behavior. $\mathrm{PhD}$ Dissertation, Texas A \& M University, College Station, Texas.

Brown, M, Ozkan, E., Raghavan, R., and Kazemi, H. 2009. Practical solutions for pressure transient responses of fractured horizontal wells in unoconvetional reservoirs. Paper SPE 125043 presented at the Annual Technical Conference and Exhibition. New Orleans, Louisiana. Society of Petroleum Engineers.

Browning, J., Tinker, S. W., Ikonnikova, S., et al. 2013. Barnett study determined full-field reserves, production forecast. Oil and Gas Journal.

Bump, A. C., and McKee, C. R. 1988. Gas-well testing in the pressure of desorption for coalbed methane and devonian Shale. SPE Formation Evaluation, SPE-15227-PA- 179-185.

Carter, R. D. 1985. Type curves for finite radial and linear gas-flow systems: Constant-terminal pressure case. SPE Journal, SPE-12917. 719-728. 
Clark, A, J., Lake, L. W., and Patzek, T. W. 2011. Production forecasting with logistic growth model. Paper SPE 144790 presented at SPE ATCE, Denver, Colorado, U.S.A. doi 10.2118/144790-MS.

Clauset, A., Shalizzi, C. R., and Newman, M. E. J. 2009. Power-Law Distributions in Empirical Data. SIAM Review 661-703. doi:10.1137/070710111.

Damoah, L. O., Mohammed, S. and Enty, G. S. 2015. Utilizing early-time boundary-dominated flow data to make an inital estimate of gas-in-place: An aid to computing material balance pseudotime. Paper SPE-178376-MS presented at the Nigeria Annual International Conference and Exhibition 1-15.

Duong. 2011. Rate-Decline Analysis for Fracture-Dominated Shale Reservoirs. SPE Reservoir Evaluation and Engineering 377-387. doi.2118.137748-PA.

El-Banbi. 1998. Analysis of Tight Gas Wells, PhD dissertation. College Station, Texas: Texas A \& M University.

Ezulike, D. O. and Dehghanpour, H. 2013. A model for simultaneous matrix depletion into natural and hydraulic fracture networks. Journal of Natural Gas Science and Engineering doi.org/10.1016/jngse.2013.11.004.

Ezulike, D. O. and Dehghanpour, H. 2015. Capturing the effects of secondary fractures on production data using flow regime equations and specialized plots: An uncertainty analysis approach. Journal of Petroleum Science and Engineering doi.org/10.1016/j.petrol.2015.11.016.

Gonzalez, M. C., Hidalgo, C. A., and Barabasi, A.,. 2008. Understanding individual human mobility pattern. Nature Publisinh Group 779-782. doi: 10.1038/nature06958.

Gupta, I., Rai, C., Devegowda, D., et al. 2019. Haynesville shale: Predicting long-term production and residual analysis to identify well inteference and fracture hits. SPE Reservoir Evaluation \& Engineering 1-11.

Hubbert, M. K. 1965. Nuclear Energy and Fossil Fuels. American Petroleum Institute API-56-007.

Ilk, D., Rushing, J. A., Perego, A. D., et al. 2008. Exponential Vs Hyperbolic Decline in Tight Gas Snads - Understanding the Origin and Implications for Reserve Estimates Using Arps Decline Curves. Paper SPE 116731 presented at the SPE Annual Technical Conference and Exhibition, Denver, Colorado, U.S.A., doi: 10.2118/116731-MS.

Javadpour, F., Fischer, D. and Unsworth, M. 2007. Nanoscale gas flow in shale gas sediments. JCPT 9(4): 451-462.

Joshi, K. and Lee, J. 2013. Comparison of various deterministic forecasting techniques in shale gas reservoirs. SPE Hydraulic Fracturing Technology Conference. Woodland, Texas, U.S.A. doi: 10.2118/163870-MS. 
Kanfar, M. S. and Wattenbarger, R. A. 2012. Comparison of empirical decline curve methods for shale gas wells. SPE Canadian Unconventional Resources Conference . Calgary, Canada: Society of Petroleum Engineers. 1-12.

Lee, W. J. and Sidle, R. E. 2010. Gas reserve estimation in resource plays. Paper SPE 130102 at the SPE Middle East Unconventional Gas. Pittsburg, Pennsylvania: Society of Petroleum Engineers.

Male, F. 2019. Using a segregated flow model to forecast production of oil, gas, and water in shale oil plays. J. Petr. Sci. Eng. 180, 48-61.

Miao, Y., Li, X., John, L., et al. 2018. A New Rate-Decline Analysis of Shale Gas Reservoirs: Coupling the Self-Diffusion and Surface Charateristics . J. Petr. Sci. Eng. 163, 166-176.

Miao, Y., Zhao, C. and Zhou, G. 2020. New rate-decline forecast approach for low-permeability gas reservoirs with hydraulic fracturing treatments. Journal of Petroleum Science and Engineering doi.org/10.1016/j.petrol.2020.107112.

Mirzaei, M. and Cipolla, C. L. 2012. A workflow for modeling and simulation of hydraulic fractures in unconvetional gas reservoirs. SPE Middle East Unconventional Gas Conference and Exhibition. Abu Dhabi, U.A.E: Society of Petroleum Engineers.

Mohammed, S. and Enty, G. 2013. Analysis of gas well production data using flowing material balance method. Paper SPE-167504-MS presented at the Nigeria Annual International Conference and Exhibition 1-22.

Moridis, G. J., and Blasingame, T. A. and Freeman, C. M. 2010. Analysis of mechanisms of flow in fracturedtTight-gas and shale-gas reservoirs. SPE Latin American \& Caribbean Petroleum Engineering Conference 1-22.

Ozcan, O, Sarak, H., Ozkan, E., et al. 2014. A trilinear flow model for a fractured horizontal well in a fractal unconventional reservoirs. Paper SPE-170971-MS presented at the SPE ATCE. Amsterdam, The Netherlands: Society of Petroleum Engineers. 1-19.

Ozkan, E., Brown, M, Raghavan, R. and Kazemi, K. 2009. Comparison of fractured horizontalwell perfoamnce in conventional and unconventional reservoirs. SPE Western Regional Meeting. San, Jose, California,: Society of Petroleum Engineers.

Passey, Q. R., Bohacs, K. M. and Esch, W. L., et al. 2010. From oil-prone source rock to gasproducing shale reservoir- Geological and petrophysical charaterization of unconventional shale reservoirs. SPE 131350 presented at the CPS/SPE Internation Oil and Gas Conference and Exhibition. Beijing, China: Society of Petroleum Engineers.

Patzek, T. W., Male, F., Marder, M. 2013. Gas production in the Barneet Shale obeys a simple scaling theory. PNAS, U. S. A. 110 (49), 19731-19736.

Raghavan, R. and Chen, C. 2013a. Fractional diffusion in rocks produced by horizontal wells with multiple, transverse hydraulic fractures of finite conductivity. Journal of Petroleum Science and Engineering. 133-143. 
Raghavan, R. and Chen, C. 2013b. "Fractured-well performance under anomalous diffusion. SPE Reservoir Evaluation \& Engineering 16(3): 237-245.

Raghavan, R. 2011. Fractional derivatives: Application to transient flow. Journal of Petroleum Science and Engineering 7-13.

Smolinsky, L. 2017. Discrete Power Law with Exponential Cutoff and Lotka's Law. Journal of the Associations for Information Science and Technology 1-4. 10.1002/asi.23763.

Valko. 2009. Assigning value to stimulation in the Barneet shale: A simultaneous analysis of 7000 plus production histories and well completion records." Paper SPE 119369 presented at the SPE Hydraulic Fracturing Technology Conference, The Woodlands, Texas, U. S. A. doi: 10.2118/19369-MS.

Valko, P. P. and Lee, W. J. 2010. A Better Way to Forecast Production from Unconvetional Gas Wells. Paper SPE 134231 presented at the SPE ATCE, Florence, Italy. doi: 10.2118/134231-MS.

Wang, K., Li, H., Wang, J., et al. 2017. Predicting production and estimated ultimate recoveries for shale gas wells: A new methodology approach. Applied Energy.

Wattenbarger, R. A. 2007. Some Reservoir Performance Aspects of Uncontional Gas Production. Private Conference Presentation.

Wattenbarger, R. A., El-Banbi, A. H., Villegas, M., et al. 1998. Production analysis of linear flow into fractured tight gas wells. Paper SPE-33931-MS presented at the SPE Rocky Mountain Regional/Low-Permeability Reservoir Symposium. Denver, Colorado, U. S. A.: Society of Petroleum Engineers.

Xue, H., Li, Y., Cai, H., et al. 2018. Productivity analysis and numerical simulation for fractured wells in unconventional gas reservoirs. Exergy for A Better Environment and Improved Sustainability 1. Green Energy and Technology. Springer, Cham https://doi.org/10.1007/978-3-319-62572-0_3.

Yu, S. Y. and Yao, J. 2013. Unconventional gas reservoir engineering. Petroleum Industry Press.

Zuo, L., Yu, W. and Wu, K. 2016. A fractional decline curve analysis model for shale gas reservoirs. Applied Energy 163: 140-148. doi.org/10.1016/j.coal.2016.07.006. 